\title{
A Quasi-Orthogonal Group Space-time Architecture to Achieve a Better Diversity-Multiplexing Tradeoff*
}

\author{
Lin Dai, Member, IEEE, Sana Sfar, Student Member, IEEE, and Khaled B. Letaief, Fellow, IEEE \\ Center for Wireless Information Technology \\ Electrical and Electronic Engineering Department \\ The Hong Kong University of Science \& Technology \\ Clear Water Bay, HONG KONG
}

\begin{abstract}
Most existing MIMO (Multiput-Input Multiput-Output) schemes optimize only either the diversity gain or the multiplexing gain. To obtain a good tradeoff between these two, the Quasi-Orthogonal Group Space-Time (QoGST) architecture is proposed, wherein the transmit stream is subgrouped but encoded via an inter-group space-time block encoder, with group interference suppression at the receiver. This paper also considers another combined space-time coding and layered space-time architecture, which we refer to as Group Layered Space-Time (GLST), where space-time block coding is employed within each group. Under the assumption of Rayleigh fading and a prior perfect channel state information at the receiver, a performance analysis will demonstrate that both QoGST and GLST can achieve a good diversity-multiplexing tradeoff. QoGST is even superior to GLST. Simulation results will validate our analysis and further show that compared to the existent Layered Space-Time Block Code (LSTBC) scheme, both QoGST and GLST can achieve a significant performance gain.
\end{abstract}

Keywords: Group detection, MIMO systems, Space-time block coding, Layered space-time, Quasi-orthogonal group space-time, Diversity-multiplexing tradeoff.

Corresponding Author:

Lin Dai

Email: eedailin@ust.hk

Tel:/Fax: (852) 2358 7086/1485

URL: http://ihome.ust.hk/ eedailin

\footnotetext{
* This work is supported in part by the Hong Kong Research Grant Council under Grant No. HKUST6030/01E.
} 


\section{Introduction}

MIMO (Multiple-Input Multiple-Output) systems have shown their ability in providing great performance improvements over the SISO (Single-Input Single-Output) systems thanks to their higher spectral efficiency [1]. It has been well understood that multiple antennas can not only be used to achieve diversity gain [2], but also as an effective way to increase the degrees of freedom of the channel [3]. With multiple antennas at both the transmitter and receiver, independent information streams can be transmitted through the parallel spatial channels so that the data rate is increased. Therefore, a MIMO system can provide both diversity gain and multiplexing gain [4].

In order to reveal the relationship between these two gains, Zheng and Tse proposed a powerful tool known as diversity-multiplexing tradeoff function [4]. It is found that a higher spatial multiplexing gain comes at the price of sacrificing diversity and vice versa. An optimal diversity-multiplexing tradeoff curve is characterized in [4] and is shown to be achievable by Gaussian random codes. Zheng and Tse also analyzed the tradeoff functions of some existing MIMO schemes and found that most of them aim at achieving either maximum diversity gain or maximum multiplexing gain. For example, space-time codes (STC) (including space-time block codes (STBC) [5-6] and space-time trellis codes (STTC) [7]) are carefully designed to achieve the full diversity order, but no multiplexing gain can be obtained. Layered space-time (LST) such as VBLAST [8] can achieve maximum multiplexing gain but with a very low diversity gain. Actually, it is shown in [4] that no existing scheme, except for the Alamouti's scheme [5] with 2 transmit antennas and 1 receive antenna and DBLAST [9] employing an MMSE decoder ${ }^{1}$, can achieve this optimal tradeoff.

There have been some efforts on explicit code construction to achieve the optimal tradeoff. [10] developed a structured coding scheme for two-transmit two-receive antenna systems with code duration two which has been shown to be able to achieve the full diversity-multiplexing frontier. [11] constructed some explicit optimal permutation codes for a parallel channel with two diversity branches. In [12], full-rate-full-diversity codes are proposed based on LCF coding and ML decoding. [13] further provided a general framework for constructing the optimal coding/decoding schemes for delay limited MIMO fading channels and claimed that their LAttice Space-Time (LAST) codes can achieve the optimal diversity-multiplexing tradeoff under generalized minimum Euclidean distance

\footnotetext{
${ }^{1}$ Here it is assumed that the overhead that is required to start the DBLAST processing is ignored.
} 
lattice decoding.

Another class of designs focuses on signal processing instead of code construction. [14] proposed a combined array processing and space-time coding architecture, in which the transmit stream is partitioned into different groups and in each group STC is applied. At the receiver, group interference suppression is adopted, where each individual STC is decoded by suppressing the signals transmitted from other groups. This combination of STC and LST provides much better multiplexing gain than STC with lower decoding complexity. At the same time, it achieves a much higher diversity gain than LST. Unlike the work in [10-13], which approach the optimal diversity-multiplexing tradeoff at a cost of high complexity, [14] shows how to trade off between diversity gain and multiplexing gain by virtue of group detection with very low complexity. [15-17] further developed this architecture. In [15] and [16], Alamouti's scheme and variable rate STBC were adopted as the component encoder, respectively, and the transmit power was optimized to minimize the average FER or BER. [17] focused on the receiver design and proposed an optimal decoding order and a computationally efficient hard-decision iterative decoding algorithm.

In [14], the substreams of each group are encoded independently and no special transmit design is adopted to suppress the interference among the groups. At the receiver, space-time decoding is performed for each group by assuming that the interference has been suppressed by virtue of a group detector. That is, in [14] (including [15-17]), group detection is performed first followed by space-time decoding. Therefore, the overall performance is limited by the group detection step.

This paper further presents a novel group space-time architecture, which we shall refer to as Quasi-Orthogonal Group Space-time (QoGST). At the transmitter, all the groups are encoded together via an inter-group STBC. To keep the same spectral efficiency as [14], we assume that in each group no space-time coding is adopted. Particularly, at each time slot $t$, we regard the transmit vector of each group as one symbol and apply STBC to all the transmit vectors. It can be seen that with this inter-group STBC, the interference among groups can be effectively suppressed because of the orthogonal nature of STBC. Therefore, QoGST should have a better interference suppressing capability. However, it should be pointed out that the encoded vectors of each group are not strictly orthogonal to each other. Instead, they are orthogonal only when the group size is one. This is why the proposed scheme is referred to as "quasi-orthogonal". This should be distinguished from [18] which aims at constructing a kind of rate one quasi-orthogonal STBC by building a $k \times k$ code matrix from 
two $\frac{k}{2} \times \frac{k}{2}$ matrices.

At the receiver, and in contrast to the detector used in [14-17], space-time block decoding is performed before group detection is applied. Specifically, for the case of $m$ transmit and $n$ receive antennas during $T$ time slots, the linear nature of STBC can be exploited to obtain an equivalent $T n \times m$ channel [19]. Group detection is then applied based on this equivalent channel. It can be seen that after decoding, the receive dimensions increase from $n$ to Tn and thus much better performance can be achieved by group detection. In this paper, we shall always assume that this novel detector is adopted instead of the one used in [14-17]. For the sake of comparison, the structure that combines the proposed detector and the transmission structure proposed in [14] with STBC in each group is considered in this paper. Such combined structure, which we refer to as Group Layered Space-Time (GLST), should be distinguished from the Layered Space-Time Block Code (LSTBC) proposed in [15], as we adopt a different detection methodology.

The performance of our proposed QoGST and GLST, is evaluated in terms of the diversity-multiplexing tradeoff function. To do so, we first obtain the equivalent channel models of GLST and QoGST, respectively, by virtue of the linear structure of STBC. For $m$-transmit- $n$-receive-T-coding-length GLST and QoGST, we derive the tradeoff function of an $m$-transmit-Tn-receive system over Rayleigh slow fading channels with group zero-forcing (GZF) detection since their equivalent channels are both of $T n \times m$ dimensions. However, it is very difficult to get the exact tradeoff function of both GLST and QoGST in general. Therefore, we resort to tradeoff bounds. Particularly, we obtain the lower and the upper bound tradeoff functions of GLST and QoGST. It is found that the lower bound tradeoff of QoGST is usually better than the upper bound tradeoff of GLST, which implies that QoGST has a better diversity-multiplexing tradeoff than GLST. Simulation results will further validate our analysis and show that compared to the existent LSTBC scheme, both QoGST and GLST can achieve a significant performance gain. Throughout the paper, we assume Rayleigh fading and a prior perfect knowledge of the channel at the receiver.

This paper is organized as follows. In Section II, we provide our channel model and briefly present the group detection scheme. In Section III, we introduce the transmitter and receiver design of GLST, and then QoGST. Section IV presents the performance analysis, which is evaluated in terms of the diversity-multiplexing tradeoff function. Simulation results are given in Section V. Finally, Section VI summarizes and concludes this paper. 


\section{Channel Model and Group Detection}

We consider a wireless link with $m$ transmit and $n$ receive antennas, which we refer to as $(m, n)$. At each time slot $t$, the encoded and modulated signal $x_{t}^{i}$ is transmitted through transmit antenna $i$, $1 \leq i \leq m$. We assume that the channel remains constant within a block of $L$ symbols. Let $h_{i j}$ denote the complex path gain from transmit antenna $j$ to receive antenna $i$, which is modeled as samples of independent complex Gaussian random variables with mean zero and variance 0.5 per dimension. We also assume perfect channel knowledge at the receiver side only, through the use of training sequences.

Let $(\cdot)^{\prime}$ denote the transpose operator. The discrete received complex signal vector can now be written as

$$
\mathbf{y}_{\mathbf{t}}=\sqrt{\frac{S N R}{m}} \mathbf{H x}_{\mathbf{t}}+\mathbf{z}_{\mathbf{t}},
$$

where $\mathbf{x}_{\mathbf{t}}=\left[x_{t}^{1}, x_{t}^{2}, \ldots, x_{t}^{m}\right]^{\prime}$ and $\mathbf{y}_{\mathbf{t}}=\left[y_{t}^{1}, y_{t}^{2}, \ldots, y_{t}^{n}\right]^{\prime}$. The additive noise $\mathbf{z}_{\mathbf{t}}$ has i.i.d. entries $\mathbf{z}_{t}^{i}$, $i=1, \ldots, n$, which are all Gaussian complex random variables with mean zero and unit variance. Also SNR is the average signal-to-noise ratio at each receive antenna.

Assume that the transmit signals are divided into $G$ groups, $\mathcal{G}_{1}, \mathcal{G}_{2}, \ldots, \mathcal{G}_{G}$, with group size $\left|\mathcal{G}_{i}\right|$, $i=1, \ldots, G$. Then, (1) can be written as

$$
\mathbf{y}_{\mathbf{t}}=\sqrt{\frac{S N R}{m}}\left[\mathbf{H}_{\mathcal{G}_{1}}, \mathbf{H}_{\mathcal{G}_{2}}, \ldots, \mathbf{H}_{\mathcal{G}_{G}}\right] \cdot\left[\begin{array}{c}
\mathbf{s}_{\mathbf{t}}^{\mathbf{1}} \\
\mathbf{s}_{\mathbf{t}}^{\mathbf{2}} \\
\vdots \\
\mathbf{s}_{\mathbf{t}}^{\mathbf{G}}
\end{array}\right]+\mathbf{z}_{\mathbf{t}},
$$

where $\mathbf{s}_{\mathbf{t}}^{\mathbf{i}}$ is the transmit vector of group $\mathcal{G}_{i}$ at time slot $t, t=1, \ldots, L$ and $i=1, \ldots, G . \mathbf{H}_{\mathcal{G}_{i}}$ is the $n \times\left|\mathcal{G}_{i}\right|$ channel matrix of group $\mathcal{G}_{i}, \quad i=1, \ldots, G$.

When group detection is adopted, two types of receiver can be deployed: Group Zero-Forcing (GZF) and Group Successive Interference Cancellation (GSIC). With GZF, groups are detected independently. In particular, at time slot $t$, group $\mathcal{G}_{i}$ is assumed to be detected. Then, the interference from the other groups $\mathcal{G}_{1}, \ldots, \mathcal{G}_{i-1}, \mathcal{G}_{i+1}, \ldots, \mathcal{G}_{G}$ should be nulled out using an orthogonal projection. To 
obtain the projection matrix, we partition $\mathbf{H}$ into $\mathbf{H}=\left[\mathbf{H}_{\mathcal{G}_{i}}, \mathbf{H}_{\overline{\mathcal{G}_{i}}}\right]$, where $\mathbf{H}_{\overline{\mathcal{G}_{i}}}$ includes the columns of $\mathbf{H}$ corresponding to all the groups except $\mathcal{G}_{i}$. The projection matrix $\mathbf{P}_{\mathcal{G}_{i}}$ is then defined as [20]

$$
\mathbf{P}_{\mathcal{G}_{i}}=\mathbf{I}_{\mathbf{n}}-\mathbf{H}_{\overline{\mathcal{G}}_{i}}\left(\mathbf{H}_{\mathcal{G}_{i}}^{+} \mathbf{H}_{\overline{\mathcal{G}}_{i}}\right)^{-1} \mathbf{H}_{\overline{\mathcal{G}}_{i}}^{+},
$$

where $(\cdot)^{+}$denotes the complex conjugate transpose. Therefore, using the transformation $\mathbf{W}_{\mathbf{i}}=\mathbf{H}_{\mathcal{G}_{i}}^{+} \mathbf{P}_{\mathcal{G}_{i}}$ on $\mathbf{y}_{\mathbf{t}}$, we have

$$
\tilde{\mathbf{y}}_{\mathbf{t}}^{\mathbf{i}}=\mathbf{W}_{\mathbf{i}} \mathbf{y}_{\mathbf{t}}=\sqrt{\frac{S N R}{m}} \mathbf{H}_{\mathcal{G}_{i}}^{+} \mathbf{P}_{\mathcal{G}_{i}} \mathbf{H}_{\mathcal{G}_{i}} \mathbf{s}_{\mathbf{t}}^{\mathbf{i}}+\tilde{\mathbf{z}}_{\mathbf{t}}=\sqrt{\frac{S N R}{m}} \mathbf{Q}_{\mathcal{G}_{i}}^{-1} \mathbf{s}_{\mathbf{t}}^{i}+\tilde{\mathbf{z}}_{\mathbf{t}},
$$

where

$$
\mathbf{Q}_{\mathcal{G}_{i}}^{-1}=\mathbf{H}_{\mathcal{G}_{i}}^{+} \mathbf{P}_{\mathcal{G}_{i}} \mathbf{H}_{\mathcal{G}_{i}} .
$$

It turns out that $\mathbf{Q}_{\mathcal{G}_{i}}=\left[\left(\mathbf{H}^{+} \mathbf{H}\right)^{-1}\right]_{\left|\mathcal{G}_{i}\right| \times\left|\mathcal{G}_{i}\right|}$ is the $\left|\mathcal{G}_{i}\right| \times\left|\mathcal{G}_{i}\right|$ diagonal submatrix of $\left(\mathbf{H}^{+} \mathbf{H}\right)^{-1}$ and the noise $\tilde{\mathbf{z}}_{\mathbf{t}}$ has covariance $\mathbf{Q}_{\mathcal{G}_{i}}^{-1}$. The transmit symbols of group $\mathcal{G}_{i}$ at time slot $t$ can then be decoded using MLD based on $\tilde{\mathbf{y}}_{\mathbf{t}}^{\mathbf{i}}$ as follows:

$$
\hat{\mathbf{s}}_{\mathbf{t}}^{\mathbf{i}}=\arg \min _{\mathbf{s}_{\mathbf{i}}^{\mathbf{i}} \in \Omega}\left(\mathbf{r}_{\mathbf{t}}^{\mathbf{i}}\right)^{+} \mathbf{Q}_{\mathcal{G}_{i}} \mathbf{r}_{\mathbf{t}}^{\mathbf{i}},
$$

where $\Omega$ is the constellation set and

$$
\mathbf{r}_{\mathbf{t}}^{\mathbf{i}}=\tilde{\mathbf{y}}_{\mathbf{t}}^{\mathbf{i}}-\sqrt{\frac{S N R}{m}} \mathbf{Q}_{\mathcal{G}_{i}}^{-1} \mathbf{s}_{\mathbf{t}}^{\mathbf{i}} .
$$

When GSIC is adopted, the channel matrix $\mathbf{H}$ is updated by eliminating the columns corresponding to $\mathcal{G}_{1}, \mathcal{G}_{2}, \ldots, \mathcal{G}_{i-1}$ before detecting $\mathcal{G}_{i}$. After obtaining $\hat{\mathbf{s}}_{\mathbf{t}}^{\mathrm{i}}$, the interference introduced by $\mathcal{G}_{i}$ should be subtracted from $\mathbf{y}_{\mathbf{t}}$. The remaining process is similar to GZF.

Throughout this paper, we denote by $(\cdot)^{*}$ and $\operatorname{det}(\cdot)$ the conjugate and the determinant operators, respectively. $\mathbf{I}_{\mathbf{m}}$ and $\mathbf{0}_{m \times n}$ represent an $m \times m$ identity matrix and an $m \times n$ zero matrix, respectively. For an arbitrary matrix $\mathbf{A}, a_{i j}$ refers to its element at the $i^{\text {th }}$ row and the $j^{\text {th }}$ column, and $(\mathbf{A})_{n \times n}$ to its $n \times n$ diagonal submatrix. When $\mathbf{A}$ is a Hermitian positive definite matrix, we shall write it as $\mathbf{A}>\mathbf{0}$. If $\mathbf{A}$ and $\mathbf{B}$ are both Hermitian matrices and we have $\mathbf{A}-\mathbf{B}>\mathbf{0}$, we write it as $\mathbf{A}>\mathbf{B}$. Finally, $\overline{\mathbf{S}}$ shall represent the complement of a set $\mathbf{S}$ with the length $|\mathbf{S}|$. 


\section{Combined STBC and LST}

We begin by presenting GLST, and then provide the details of the QoGST architecture.

\section{A. GLST}

\section{A.1 Transmitter}

As shown in Fig. 1, all the $m$ transmit antennas are partitioned into $G_{m}$ groups, respectively, comprising $m_{1}, m_{2}, \ldots, m_{G}$ antennas with $\sum_{i=1}^{G_{m}} m_{i}=m$. A block of input bits $\left\{b_{i}\right\}_{i=1 \ldots K}$ with length $K$ is divided into $G_{b}$ groups, $\mathcal{G}_{1}, \mathcal{G}_{2}, \ldots, \mathcal{G}_{G_{b}}$, and in each group, $\mathbf{b}_{\mathbf{i}}=\left[b_{i, 1}, b_{i, 2}, \ldots, b_{i, \mid \mathcal{G}_{i}}\right], i=1, \ldots, G_{b}$, is then encoded by a component space-time block code $S T B C_{i}$ associated with $m_{i}$ transmit antennas. In GLST, it is required that $G_{m}=G_{b}=G$. For simplicity, here we do not consider the case of variable-rate STBC. Therefore, we assume that all the component codes $S T B C_{i}, i=1, \ldots, G$, have the same code length $T$, and we have $m_{i}=g_{m}$ and $\left|\mathcal{G}_{i}\right|=g_{b}$ for $i=1, \ldots, G$. Then, the output $m \times T$ codeword matrix $\mathbf{X}$ over a block of $T$ symbol intervals can be written as

$$
\mathbf{X}=\left[\begin{array}{ccc}
x_{1}^{1} & \cdots & x_{T}^{1} \\
\vdots & \ddots & \vdots \\
x_{1}^{m} & \cdots & x_{T}^{m}
\end{array}\right]=\left[\begin{array}{ccc}
\mathbf{s}_{1}^{1} & \cdots & \mathbf{s}_{T}^{1} \\
\vdots & \ddots & \vdots \\
\mathbf{s}_{1}^{G} & \cdots & \mathbf{s}_{T}^{G}
\end{array}\right]=\left[\begin{array}{c}
\mathbf{S}_{1} \\
\vdots \\
\mathbf{S}_{G}
\end{array}\right],
$$

where $\mathbf{S}_{i}=\left[\mathbf{s}_{1}^{i}, \cdots, \mathbf{s}_{T}^{i}\right]$ is the $g_{m} \times T$ codeword matrix of group $\mathcal{G}_{i}, i=1, \ldots, G$.

An $m$-antenna- $T$-time-slot- $K$-symbol STBC $\mathcal{O}_{x}$ can be represented as [23]

$$
\mathcal{O}_{x}=\left[\mathbf{A}_{1} \mathbf{x}+\mathbf{B}_{1} \mathbf{x}^{*}, \mathbf{A}_{2} \mathbf{x}+\mathbf{B}_{2} \mathbf{x}^{*}, \ldots, \mathbf{A}_{T} \mathbf{x}+\mathbf{B}_{T} \mathbf{x}^{*}\right]
$$

where $\mathbf{x}$ is an $K \times 1$ complex variable vector and $\mathbf{A}_{t}, \mathbf{B}_{t}$ are constant coefficient matrices in $\mathcal{R}^{m \times K}, t=1, \ldots, T$. The matrix $\mathcal{O}_{x}$ is called $[m, T, K]$ STBC for short in the following. Therefore, $\mathbf{S}_{i}$ can be written as

$$
\mathbf{S}_{i}=\left[\mathbf{A}_{1}^{i} \mathbf{b}_{i}, \ldots, \mathbf{A}_{T}^{i} \mathbf{b}_{i}\right]+\left[\mathbf{B}_{1}^{i} \mathbf{b}_{i}^{*}, \ldots, \mathbf{B}_{T}^{i} \mathbf{b}_{i}^{*}\right]
$$

for $i=1, \ldots, G$, where $\mathbf{A}_{t}^{i}, \mathbf{B}_{t}^{i}$ are constant coefficient matrices in $\mathcal{R}^{g_{m} \times g_{b}}, t=1, \ldots, T$.

It can be seen that in this transmit architecture, the bit streams of each group are space-time coded. 
Therefore, a higher diversity gain can be achieved compared to the conventional LST. Besides, the multiplexing gain is higher than the conventional STBC due to the use of multiple group transmission. We can thus conclude that this transmit scheme offers a good tradeoff between the diversity gain and multiplexing gain.

\section{A.2 Receiver}

The detector presented in [14-17] is to suppress signals transmitted from other groups of antennas by virtue of a group detector first, and then perform space-time decoding for the desired group. Particularly, assume that GZF is adopted. At each time slot $t$, the nulling matrix $\mathbf{W}_{i}=\mathbf{H}_{\mathcal{G}_{i}}^{+} \mathbf{P}_{\mathcal{G}_{i}}$ for group $\mathcal{G}_{i}$ can be computed, as shown in Section II. However, to decode the whole codeword $\mathbf{S}_{i}$ of group $\mathcal{G}_{i}$, an ML space-time decoder should be adopted instead of the one given by (6) as

$$
\hat{\mathbf{b}}_{i}=\arg \min _{\mathbf{b}_{i} \in \Omega} \sum_{t=1}^{T}\left(\mathbf{r}_{t}^{i}\right)^{+} \mathbf{Q}_{\mathcal{G}_{i}} \mathbf{r}_{t}^{i},
$$

where $\mathbf{r}_{\mathbf{t}}^{\mathrm{i}}$ is given by (7).

In this paper, we adopt a new detector, in which space-time decoding is performed first followed by group detection. To do so, an equivalent channel is obtained by virtue of the linear nature of STBC. GZF is then performed. Particularly, by combining (8) and (10), the received signal vector can be written as

$$
\begin{aligned}
\mathbf{Y} & =\sqrt{\frac{S N R}{m}}\left[\left[\mathbf{H}_{1} \mathbf{A}_{1}^{1}, \ldots, \mathbf{H}_{G} \mathbf{A}_{1}^{G}\right] \cdot\left[\begin{array}{c}
\mathbf{b}_{1} \\
\vdots \\
\mathbf{b}_{G}
\end{array}\right], \quad \cdots, \quad\left[\mathbf{H}_{1} \mathbf{A}_{T}^{1}, \ldots, \mathbf{H}_{G} \mathbf{A}_{T}^{G}\right] \cdot\left[\begin{array}{c}
\mathbf{b}_{1} \\
\vdots \\
\mathbf{b}_{G}
\end{array}\right]\right]+ \\
& \sqrt{\frac{S N R}{m}}\left[\left[\mathbf{H}_{1} \mathbf{B}_{1}^{1}, \ldots, \mathbf{H}_{G} \mathbf{B}_{1}^{G}\right] \cdot\left[\begin{array}{c}
\mathbf{b}_{1}^{*} \\
\vdots \\
\mathbf{b}_{G}^{*}
\end{array}\right], \cdots, \quad\left[\mathbf{H}_{1} \mathbf{B}_{T}^{1}, \ldots, \mathbf{H}_{G} \mathbf{B}_{T}^{G}\right] \cdot\left[\begin{array}{c}
\mathbf{b}_{1}^{*} \\
\vdots \\
\mathbf{b}_{G}^{*}
\end{array}\right]\right]+\mathbf{Z} .
\end{aligned}
$$

Let $\mathbf{y}_{i}$ represent the $i$-th column vector of $\mathbf{Y}$ and $\mathbf{r}=\left[\mathbf{y}_{1}^{\prime}, \mathbf{y}_{2}^{\prime}, \ldots, \mathbf{y}_{T}^{\prime}\right]^{\prime}$. Then, from (12) we have

$$
\mathbf{r}=\sqrt{\frac{S N R}{m}}\left\{\left[\begin{array}{ccc}
\mathbf{H}_{1} \mathbf{A}_{1}^{1} & \cdots & \mathbf{H}_{G} \mathbf{A}_{1}^{G} \\
\vdots & \ddots & \vdots \\
\mathbf{H}_{1} \mathbf{A}_{T}^{1} & \cdots & \mathbf{H}_{G} \mathbf{A}_{T}^{G}
\end{array}\right] \cdot\left[\begin{array}{c}
\mathbf{b}_{1} \\
\vdots \\
\mathbf{b}_{G}
\end{array}\right]+\left[\begin{array}{ccc}
\mathbf{H}_{1} \mathbf{B}_{1}^{1} & \cdots & \mathbf{H}_{G} \mathbf{B}_{1}^{G} \\
\vdots & \ddots & \vdots \\
\mathbf{H}_{1} \mathbf{B}_{T}^{1} & \cdots & \mathbf{H}_{G} \mathbf{B}_{T}^{G}
\end{array}\right] \cdot\left[\begin{array}{c}
\mathbf{b}_{1}^{*} \\
\vdots \\
\mathbf{b}_{G}^{*}
\end{array}\right]\right\}+\mathbf{v}
$$

[23] has proposed an algorithm for constructing any $[m, T, K]$ STBC that guarantees that $\mathbf{b}$ and $\mathbf{b}^{*}$ will not appear in the same time slot $t$. Under this assumption, (13) is equivalent to 


$$
\tilde{\mathbf{r}}=\sqrt{\frac{S N R}{m}}\left\{\left[\begin{array}{ccc}
\mathbf{H}_{1} \mathbf{A}_{1}^{1}+\mathbf{H}_{1}^{*} \mathbf{B}_{1}^{1^{*}} & \cdots & \mathbf{H}_{G} \mathbf{A}_{1}^{G}+\mathbf{H}_{G}^{*} \mathbf{B}_{1}^{G^{*}} \\
\vdots & \ddots & \vdots \\
\mathbf{H}_{1} \mathbf{A}_{T}^{1}+\mathbf{H}_{1}^{*} \mathbf{B}_{T}^{1^{*}} & \cdots & \mathbf{H}_{G} \mathbf{A}_{T}^{G}+\mathbf{H}_{G}^{*} \mathbf{B}_{T}^{G^{*}}
\end{array}\right] \cdot\left[\begin{array}{c}
\mathbf{b}_{1} \\
\vdots \\
\mathbf{b}_{G}
\end{array}\right]\right\}+\tilde{\mathbf{v}}=\sqrt{\frac{S N R}{m}}\left[\mathbf{H}_{\mathcal{G}_{1}}, \ldots, \mathbf{H}_{\mathcal{G}_{G}}\right] \cdot\left[\begin{array}{c}
\mathbf{b}_{1} \\
\vdots \\
\mathbf{b}_{G}
\end{array}\right]+\tilde{\mathbf{v}}
$$

where $\tilde{\mathbf{r}}=\left[\tilde{\mathbf{y}}_{1}^{\prime}, \ldots, \tilde{\mathbf{y}}_{T}^{\prime}\right]^{\prime}$ and $\tilde{\mathbf{y}}_{t}=\left\{\begin{array}{ll}\mathbf{y}_{t}, & \mathbf{B}_{t}^{i}=\mathbf{0}_{g_{m} \times g_{b}} \\ \mathbf{y}_{t}^{*}, & \mathbf{A}_{t}^{i}=\mathbf{0}_{g_{m} \times g_{b}}\end{array}, t=1, \ldots, T\right.$. Also $\tilde{\mathbf{v}}$ has covariance $\mathbf{I}_{T n}$. For any group $\mathcal{G}_{i}, \quad i=1, \ldots, G$, its corresponding subchannel matrix is given by

$$
\mathbf{H}_{\mathcal{G}_{i}}=\left[\begin{array}{c}
\mathbf{H}_{i} \mathbf{A}_{1}^{i}+\mathbf{H}_{i}^{*} \mathbf{B}_{1}^{i^{*}} \\
\vdots \\
\mathbf{H}_{i} \mathbf{A}_{T}^{i}+\mathbf{H}_{i}^{*} \mathbf{B}_{T}^{i *}
\end{array}\right]
$$

Notice that (15) is an equivalent subchannel for each group with subchannel $\mathbf{H}_{i}$. From (15), it can be also seen that the equivalent subchannels of each group are independent. This is because an independent STBC is adopted for each group in GLST. Notice that $\left\{\mathbf{A}_{t}^{i}\right\}_{t=1, \ldots, T}$ and $\left\{\mathbf{B}_{t}^{i}\right\}_{t=1, \ldots, T}$, are the coefficient matrices of $S T B C_{i}, \quad i=1, \ldots, G$. We present the following proposition.

Proposition 1: For the equivalent channel matrix of any group, $\mathbf{H}_{\mathcal{G}_{i}}=\left[\overline{\mathbf{h}}_{i, 1}, \ldots, \overline{\mathbf{h}}_{i, g_{b}}\right], \quad i=1, \ldots, G$, its column vectors are orthogonal to each other, namely,

$$
\overline{\mathbf{h}}_{i k}^{+} \overline{\mathbf{h}}_{i j}=\left\{\begin{array}{cc}
\sum_{l=1}^{g_{m}} \mathbf{h}_{i, l}^{+} \mathbf{h}_{i, l} & j=k \\
0 & \text { otherwise }
\end{array},\right.
$$

where $\mathbf{h}_{i, l}$ is the $\left((i-1) \cdot g_{m}+l\right)^{\text {th }}$ column vector of the channel matrix $\mathbf{H}, l=1, \ldots, g_{m}$ and $i=1, \ldots, G$.

Proposition 1 can be easily obtained from the properties of $\left\{\mathbf{A}_{t}^{i}\right\}_{t=1, \ldots, T}$ and $\left\{\mathbf{B}_{t}^{i}\right\}_{t=1, \ldots, T}$. Therefore, we omit the proof here.

From (14) it is clear that after obtaining this $T n \times K$ equivalent channel of GLST, the decoding process is done. Group detection can then be applied so as to get the original transmit symbols. The details have been presented in Section II and we do not repeat them here. Besides, it should be noticed that there should be some constraint on the number of receiver antennas since group detection is applied to a $\operatorname{Tn} \times K$ equivalent channel at the receiver. In particular, $T n$ should be larger than $m-g_{m}$ in order to ensure that the group detector works. 


\section{B. QoGST}

\section{B.1 Transmitter}

In GLST, the bit streams of each group are encoded separately so that the output streams $\mathbf{S}_{1}, \mathbf{S}_{2}, \ldots, \mathbf{S}_{\mathrm{G}}$ are independent of each other. No special transmit design is adopted to suppress the interference among the groups. Besides, the mapping from different groups to the transmit antennas is always fixed over all the time slots. Therefore, no interleaving gain can be achieved. In this section, we will present a new space-time architecture, in which all the groups are encoded together via an “inter-group STBC” encoder. We call it Quasi-Orthogonal Group Space-time (QoGST).

As Fig. 2 shows, the input stream $\left\{b_{i}\right\}_{i=1 \ldots K}$ and all the $m$ transmit antennas are equally partitioned into $G_{b}$ and $G_{m}$ groups with the group size $g_{b}$ and $g_{m}$, respectively, as GLST does. However, instead of being encoded separately, all the groups $\mathbf{b}_{1}, \mathbf{b}_{2}, \ldots, \mathbf{b}_{G_{b}}$ are encoded together. The design of the inter-group STBC is given by

$$
\mathbf{X}=\left[\tilde{\mathbf{A}}_{1} \mathbf{b}, \ldots, \tilde{\mathbf{A}}_{T} \mathbf{b}\right]+\left[\tilde{\mathbf{B}}_{1} \mathbf{b}^{*}, \ldots, \tilde{\mathbf{B}}_{T} \mathbf{b}^{*}\right]
$$

where

$$
\tilde{\mathbf{A}}_{t}=\left[\tilde{\mathbf{A}}_{t}^{1}, \ldots, \tilde{\mathbf{A}}_{t}^{G_{b}}\right], \quad \tilde{\mathbf{B}}_{t}=\left[\tilde{\mathbf{B}}_{t}^{1}, \ldots, \tilde{\mathbf{B}}_{t}^{G_{b}}\right]
$$

and $\quad \tilde{\mathbf{A}}_{t}^{i}=\mathbf{A}_{t}[:, i] \otimes \mathbf{I}_{g}, \quad \tilde{\mathbf{B}}_{t}^{i}=\mathbf{B}_{t}[:, i] \otimes \mathbf{I}_{g}, \quad i=1, \ldots, G_{b}$ and $t=1, \ldots, T . \quad \mathbf{A}_{t}[:, i]$ and $\mathbf{B}_{t}[:, i]$ are the $i^{t h}$ column vector of $\mathbf{A}_{t}$ and $\mathbf{B}_{t}$, respectively. $\mathbf{A}_{t}$ and $\mathbf{B}_{t}, t=1, \ldots, T$, are the coefficient matrices of a $\left[G_{m}, T, G_{b}\right]$ STBC. Here it is required that $g_{m}=g_{b}=g$.

To further illustrate this encoding process, we consider the following example. Assume that the bit streams are divided into $G_{b}=2$ groups and transmitted by $m=4$ transmit antennas over $T=2$ time slots. Obviously, we have $g=2$ and $G_{b}=G_{m}=2$. For a [2,2,2] STBC, the coefficients matrices $\mathbf{A}_{1}, \mathbf{A}_{2}, \mathbf{B}_{1}, \mathbf{B}_{2}$ are given by:

$$
\mathbf{A}_{1}=\left[\begin{array}{ll}
1 & 0 \\
0 & 1
\end{array}\right], \quad \mathbf{B}_{2}=\left[\begin{array}{cc}
0 & -1 \\
1 & 0
\end{array}\right], \quad \mathbf{A}_{2}=\mathbf{B}_{1}=\mathbf{0}_{2 \times 2}
$$

Then, the new coefficients matrices can be obtained as: 


$$
\tilde{\mathbf{A}}_{1}=\left[\begin{array}{cccc}
1 & 0 & 0 & 0 \\
0 & 1 & 0 & 0 \\
0 & 0 & 1 & 0 \\
0 & 0 & 0 & 1
\end{array}\right], \quad \tilde{\mathbf{B}}_{2}=\left[\begin{array}{cccc}
0 & 0 & -1 & 0 \\
0 & 0 & 0 & -1 \\
1 & 0 & 0 & 0 \\
0 & 1 & 0 & 0
\end{array}\right], \quad \tilde{\mathbf{A}}_{2}=\tilde{\mathbf{B}}_{1}=\mathbf{0}_{4 \times 4} .
$$

Therefore, the output codeword matrix of QoGST is given by:

$$
\mathbf{X}_{\text {QoGST }}=\left[\begin{array}{cc}
b_{1,1} & -b_{2,1}^{*} \\
b_{1,2} & -b_{2,2}^{*} \\
b_{2,1} & b_{1,1}^{*} \\
b_{2,2} & b_{1,2}^{*}
\end{array}\right] .
$$

Compared to the codeword matrix of GLST:

$$
\mathbf{X}_{G L S T}=\left[\begin{array}{cc}
b_{1,1} & -b_{1,2}^{*} \\
b_{1,2} & b_{1,1}^{*} \\
b_{2,1} & -b_{2,2}^{*} \\
b_{2,2} & b_{2,1}^{*}
\end{array}\right],
$$

it is obvious that in QoGST the mapping from the bit streams of different groups to the transmit antennas is not constant any more. Therefore, a higher diversity gain can be achieved thanks to an interleaving gain that is obtained as a result of this non-constant mapping. Besides, here STBC is applied to the transmit vectors. The interference among the groups is not independent any more and thus can be better suppressed.

\section{B.2 Receiver}

From (17), we have

$$
\begin{aligned}
& \mathbf{Y}=\sqrt{\frac{S N R}{m}}\left[\left[\mathbf{H} \tilde{\mathbf{A}}_{1}^{1}, \ldots, \mathbf{H} \tilde{\mathbf{A}}_{1}^{G_{b}}\right] \cdot\left[\begin{array}{c}
\mathbf{b}_{1} \\
\vdots \\
\mathbf{b}_{G_{b}}
\end{array}\right], \quad \cdots, \quad\left[\mathbf{H} \tilde{\mathbf{A}}_{T}^{1}, \ldots, \mathbf{H} \tilde{\mathbf{A}}_{T}^{G_{b}}\right] \cdot\left[\begin{array}{c}
\mathbf{b}_{1} \\
\vdots \\
\mathbf{b}_{G_{b}}
\end{array}\right]\right]+ \\
& \sqrt{\frac{S N R}{m}}\left[\left[\mathbf{H} \tilde{\mathbf{B}}_{1}^{1}, \ldots, \mathbf{H} \tilde{\mathbf{B}}_{1}^{G_{b}}\right] \cdot\left[\begin{array}{c}
\mathbf{b}_{1}^{*} \\
\vdots \\
\mathbf{b}_{G_{b}}^{*}
\end{array}\right], \quad \cdots, \quad\left[\mathbf{H} \tilde{\mathbf{B}}_{T}^{1}, \ldots, \mathbf{H} \tilde{\mathbf{B}}_{T}^{G_{b}}\right] \cdot\left[\begin{array}{c}
\mathbf{b}_{1}^{*} \\
\vdots \\
\mathbf{b}_{G_{b}}^{*}
\end{array}\right]\right]+\mathbf{Z} .
\end{aligned}
$$

Similarly, we can get

$$
\mathbf{r}=\sqrt{\frac{S N R}{m}}\left\{\left[\begin{array}{ccc}
\mathbf{H} \tilde{\mathbf{A}}_{1}^{1} & \cdots & \mathbf{H} \tilde{\mathbf{A}}_{1}^{G_{b}} \\
\vdots & \ddots & \vdots \\
\mathbf{H} \tilde{\mathbf{A}}_{T}^{1} & \cdots & \mathbf{H} \tilde{\mathbf{A}}_{T}^{G_{b}}
\end{array}\right] \cdot\left[\begin{array}{c}
\mathbf{b}_{1} \\
\vdots \\
\mathbf{b}_{G_{b}}
\end{array}\right]+\left[\begin{array}{ccc}
\mathbf{H} \tilde{\mathbf{B}}_{1}^{1} & \cdots & \mathbf{H} \tilde{\mathbf{B}}_{1}^{G_{b}} \\
\vdots & \ddots & \vdots \\
\mathbf{H} \tilde{\mathbf{B}}_{T}^{1} & \cdots & \mathbf{H} \tilde{\mathbf{B}}_{T}^{G_{b}}
\end{array}\right] \cdot\left[\begin{array}{c}
\mathbf{b}_{1}^{*} \\
\vdots \\
\mathbf{b}_{G_{b}}^{*}
\end{array}\right]\right\}+\mathbf{v}
$$

and 


$$
\tilde{\mathbf{r}}=\sqrt{\frac{S N R}{m}}\left\{\left[\begin{array}{ccc}
\mathbf{H} \tilde{\mathbf{A}}_{1}^{1}+\mathbf{H}^{*} \tilde{\mathbf{B}}_{1}^{1^{*}} & \cdots & \mathbf{H} \tilde{\mathbf{A}}_{1}^{G_{b}}+\mathbf{H}^{*} \tilde{\mathbf{B}}_{1}^{G_{b}{ }^{*}} \\
\vdots & \ddots & \vdots \\
\mathbf{H}^{\tilde{\mathbf{A}}_{T}^{1}}+\mathbf{H}^{*} \tilde{\mathbf{B}}_{T}^{1^{*}} & \cdots & \mathbf{H} \tilde{\mathbf{A}}_{T}^{G_{b}}+\mathbf{H}^{*} \tilde{\mathbf{B}}_{T}^{G_{b}{ }^{*}}
\end{array}\right] \cdot\left[\begin{array}{c}
\mathbf{b}_{1} \\
\vdots \\
\mathbf{b}_{G_{b}}
\end{array}\right]\right\}+\tilde{\mathbf{v}}=\sqrt{\frac{S N R}{m}}\left[\mathbf{H}_{\mathcal{G}_{1}}, \ldots, \mathbf{H}_{\mathcal{G}_{G_{b}}}\right] \cdot\left[\begin{array}{c}
\mathbf{b}_{1} \\
\vdots \\
\mathbf{b}_{G_{b}}
\end{array}\right]+\tilde{\mathbf{v}}
$$

where for $i=1, \ldots, G_{b}$,

$$
\mathbf{H}_{\mathcal{G}_{i}}=\left[\begin{array}{c}
\mathbf{H} \tilde{\mathbf{A}}_{1}^{i}+\mathbf{H}^{*} \tilde{\mathbf{B}}_{1}^{i *} \\
\vdots \\
\mathbf{H} \tilde{\mathbf{A}}_{T}^{i}+\mathbf{H}^{*} \tilde{\mathbf{B}}_{T}^{i *}
\end{array}\right]
$$

Given $\tilde{\mathbf{r}}$, group detection can then be applied.

Clearly, the equivalent channel matrix of QoGST with the element given by (26) also has $T n \times K$ dimensions. However, in contrast to GLST, here $\mathbf{H}_{\mathcal{G}_{i}}$ 's are dependent on each other. Notice that when the group size $g$ is one, QoGST is reduced to an STBC scheme and thus all $\mathbf{H}_{\mathcal{G}_{i}}$ 's are orthogonal. However, when $g$ is larger than one, $\mathbf{H}_{\mathcal{G}_{i}}$ 's are not strictly orthogonal. Actually, for any $i \neq j$, $\mathbf{H}_{\mathcal{G}_{j}}^{+} \mathbf{H}_{\mathcal{G}_{i}}$ is a matrix with zero diagonal elements instead of $\mathbf{0}_{g \times g}$. Nevertheless, compared with GLST, QoGST is expected to achieve better performance since the interference among the groups is better suppressed. We further provide the following proposition.

Proposition 2: The row vectors, $\mathbf{H}_{\mathcal{G}_{i}}[j,:], j=1, \ldots$, Tn , of any sub-channel matrix $\mathbf{H}_{\mathcal{G}_{i}}$, $i=1, \ldots, G_{b}$, are independent.

Proof: See Appendix I.

\section{Performance Evaluation}

In this section, we evaluate the performance of QoGST and GLST in terms of diversity-multiplexing tradeoff. From (14) and (25), it is clear that QoGST and GLST both have a $T n \times m$ equivalent channel. Group detection can be then applied based on the equivalent channel so as to get the original transmit symbols. This implies that the diversity gain of GLST and QoGST should be the same as the $m$-transmit $T n$-receive systems with a group detector over a channel given by (14) and (25), respectively. Therefore, before investigating the diversity-multiplexing tradeoff of GLST and QoGST, we first derive the tradeoff function of an $m$-transmit-Tn-receive system over Rayleigh quasi-static channels with GZF (we refer it to as $(m, N) \mathrm{GZF}$, where $N=T n$ ). 


\section{A. Tradeoff of $(m, N) G Z F$}

Assume that an $(m, N)$ system is given by

$$
\mathbf{y}=\sqrt{\frac{S N R}{m}} \mathbf{H b}+\mathbf{z}
$$

where the elements of $\mathbf{H}$ and $\mathbf{z}$ are all independent complex Gaussian random variables with mean zero and variance 0.5 per dimension.

From (4) we know that, for any group $\mathcal{G}_{i}, \quad i=1, \ldots, G$, we have

$$
\mathbf{y}_{\mathcal{G}_{i}}=\sqrt{\frac{S N R}{m}} \mathbf{Q}_{\mathcal{G}_{i}}^{-1} \mathbf{b}_{\mathcal{G}_{i}}+\mathbf{z}_{\mathcal{G}_{i}},
$$

where $\left\{b_{i j}\right\}_{j=1, \ldots,\left|\mathcal{G}_{i}\right|}$ are independent with the covariance matrix $\boldsymbol{\Gamma} . \mathbf{z}_{\mathcal{G}_{i}}$ has the covariance $\mathbf{Q}_{\mathcal{G}_{i}}^{-1}$. Therefore, the mutual information is given by

$$
I\left(\mathbf{b}_{\mathcal{G}_{i}} ; \mathbf{y}_{\mathcal{G}_{i}} \mid \mathbf{H}=\mathbf{Q}_{\mathcal{G}_{i}}^{-1}\right)=\log \operatorname{det}\left(\frac{\mathbf{Q}_{\mathcal{G}_{i}}^{-1}+S N R / m \cdot \mathbf{Q}_{\mathcal{G}_{i}}^{-1} \boldsymbol{\Gamma}\left(\mathbf{Q}_{\mathcal{G}_{i}}^{-1}\right)^{+}}{\mathbf{Q}_{\mathcal{G}_{i}}^{-1}}\right)=\log \operatorname{det}\left(\mathbf{I}_{\left|\mathcal{G}_{i}\right|}+\frac{S N R}{m} \boldsymbol{\Gamma}\left(\mathbf{Q}_{\mathcal{G}_{i}}^{-1}\right)^{+}\right)
$$

From [4], we know that the outage probability $P_{\mathcal{G}_{i}, \text { out }}\left(R_{\mathcal{G}_{i}}\right)=P\left[I\left(\mathbf{b}_{\mathcal{G}_{i}} ; \mathbf{y}_{\mathcal{G}_{i}} \mid \mathbf{H}=\mathbf{Q}_{\mathcal{G}_{i}}^{-1}\right)<R_{\mathcal{G}_{i}}\right]$ is given by

$$
P_{\mathcal{G}_{i}, \text { out }}\left(R_{\mathcal{G}_{i}}\right) \doteq P\left[\log \operatorname{det}\left(\mathbf{I}_{\left|\mathcal{G}_{i}\right|}+S N R \cdot \mathbf{Q}_{\mathcal{G}_{i}}^{-1}\right)<R_{\mathcal{G}_{i}}\right]=P\left[\log \prod_{j=1}^{\left|\mathcal{G}_{i}\right|}\left(1+S N R \cdot \lambda_{j}\right)<R_{\mathcal{G}_{i}}\right]
$$

where $\doteq$ is defined in [4], specifically, $f(S N R) \doteq S N R^{b}$ means that $\lim _{S N R \rightarrow \infty} \frac{\log f(S N R)}{\log S N R}=b$. Also $\left\{\lambda_{j}\right\}_{j=1, \ldots, \mathcal{G}_{i} \mid}$ are the ascending ordered eigenvalues of $\mathbf{Q}_{\mathcal{G}_{i}}^{-1}$.

Theorem 1: $\mathbf{Q}_{\mathcal{G}_{i}}^{-1} \sim W_{\left|\mathcal{G}_{i}\right|}\left(N-\left|\overline{\mathcal{G}_{i}}\right|\right)$ and the joint probability density function (pdf) of $\left\{\lambda_{j}\right\}_{j=1, \ldots,\left|\mathcal{G}_{i}\right|}$ is given by

$$
p\left(\lambda_{1}, \lambda_{2}, \ldots, \lambda_{\mathcal{G}_{i} \mid}\right)=\mathrm{K}_{\left|\mathcal{G}_{i}\right|, N-\left|\overline{\mathcal{G}}_{i}\right|} \prod_{j=1}^{\left|\mathcal{G}_{i}\right|} \lambda_{j}^{N-\left|\overline{\mathcal{G}}_{i}\right|-\left|\mathcal{G}_{i}\right|} \prod_{j<k}\left(\lambda_{j}-\lambda_{k}\right)^{2} e^{-\sum_{j=1}^{\left|\mathcal{G}_{\mid}\right|} \lambda_{j}} .
$$

Proof: See Appendix II.

In [4], it has been proved that for an $n \times m$ Rayleigh channel $\mathbf{H}, \mathbf{H}^{+} \mathbf{H} \sim W_{m}(n)$. Then, the diversity-multiplexing tradeoff $d_{\text {out }}(r) \doteq-\log P_{\text {out }}(R) / \log S N R=(m-r)(n-r)$, where $r$ is the 
multiplexing gain and $r=R / \log (S N R)$. Therefore, by applying a similar approach as the one in [4], it can be derived that

$$
d_{\mathcal{G}_{i}, \text { out }}\left(r_{\mathcal{G}_{i}}\right)=\left(N-m+\left|\mathcal{G}_{i}\right|-r_{\mathcal{G}_{i}}\right)\left(\left|\mathcal{G}_{i}\right|-r_{\mathcal{G}_{i}}\right)
$$

where $r_{\mathcal{G}_{i}}=R_{\mathcal{G}_{i}} / \log (S N R)$ is the spatial multiplexing gain of group $\mathcal{G}_{i}$.

Theorem 2: When the block length $L \geq N-\left|\overline{\mathcal{G}}_{x}\right|+\left|\mathcal{G}_{x}\right|-1 \quad$ (where $\mathcal{G}_{x}=\arg \max _{i=1, \ldots, G}\left|\mathcal{G}_{i}\right|$ ), the diversity-multiplexing tradeoff of an m-transmit- $N$-receive system with GZF is given by

$$
d(r)=\left\{\min _{i=1, \ldots G}\left\{d_{\mathcal{G}_{i}, \text { out }}\left(r_{\mathcal{G}_{i}}\right)\right\} ; \sum_{i=1}^{G} r_{\mathcal{G}_{i}}=r\right\}
$$

where $d_{\mathcal{G}_{i}, \text { out }}\left(r_{\mathcal{G}_{i}}\right)$ is defined by (32).

Proof: See Appendix III.

The tradeoff function presented in Theorem 2 clearly depends on the rate $R_{\mathcal{G}_{i}}$ and size $\left|\mathcal{G}_{i}\right|$ of each group. If all $G$ groups are assumed to be allocated the same rate with the same size $g$, the diversity-multiplexing tradeoff is given by

$$
d_{\text {eqr }}(r)=(N-m+g-r / G)(g-r / G) .
$$

From (34), it can be seen that $(m, N)$ GZF can achieve the maximum diversity gain $g(N-m+g)$. With an increasing group size $g$, a better diversity-multiplexing tradeoff can be achieved but at the cost of higher complexity. ${ }^{2}$

\section{B. Tradeoff of GLST}

The equivalent $\operatorname{Tn} \times m$ channel of GLST has been given by (14). It can be seen that although the row vectors of $\mathbf{H}, \mathbf{h}_{i}, i=1, \ldots, n$ are independent Gaussian distributed vectors, the row vectors of $\mathbf{H}_{\mathcal{G}_{i}}$ (given by (15)) are not i.i.d. any more. Hence, Theorem 1 cannot be directly applied. Here, we resort to the upper and lower bounds of the tradeoff function.

Theorem 3: The diversity-multiplexing tradeoff of GLST is bounded by

$$
d_{\text {lower_GLST }}(r) \leq d_{\text {GLST }}(r) \leq d_{\text {upper_GLST }}(r),
$$

where

\footnotetext{
${ }^{2}$ In the following, equal rate allocation is assumed in QoGST, GLST and LSTBC.
} 


$$
d_{\text {upper_GLST }}(r)=g_{m} n(1-\operatorname{Tr} / K) \text { and } d_{\text {lower_GLST }}(r)=(x-\operatorname{Tr} / G)\left(g_{b}-\operatorname{Tr} / G\right) \text {, }
$$

with $x=\min \left(n, T n-K+g_{b}\right)$.

\section{Proof: See Appendix IV.}

Orthogonal design and VBLAST can both be regarded as special cases of GLST. When $G=m=K=n$ and $g_{b}=g_{m}=1$, GLST is reduced to VBLAST. Notice here that $n=K$ and $T=1$. Therefore, $x=T n-K+g_{b}=1$. From Appendix IV, we know that the lower bound can be achieved only when $x=T n-K+g_{b}$. Therefore, we have $d_{G L S T}(r)=1-r / K \equiv d_{V B L A S T}(r)$, which is exactly the same as the one presented in [4]. On the other hand, we also show in Appendix IV that when there is only one group, the upper bound can be achieved, i.e., $d_{G L S T}(r)=m n(1-\operatorname{Tr} / K)$. In this case, GLST is reduced to orthogonal design and obviously we have ${ }^{3} d_{G L S T}(r)=d_{\text {ortho }}(r)=m n(1-\operatorname{Tr} / K)$.

\section{Tradeoff of QoGST}

The equivalent $T n \times m$ channel of QoGST has been given by (25). Since $\mathbf{H}_{\mathcal{G}_{i}}$ 's are not independent, $\mathbf{P}_{\mathcal{G}_{i}}$ is dependent on $\mathbf{H}_{\mathcal{G}_{i}}, i=1, \ldots, G_{b}$. Hence, Theorem 1 cannot be applied and we also resort to the upper and lower bounds of the tradeoff function.

Theorem 4: The diversity-multiplexing tradeoff of QoGST is bounded by

$$
d_{\text {lower_QoGST }}(r) \leq d_{\text {QoGST }}(r) \leq d_{\text {upper_QoGST }}(r)
$$

where

$$
d_{\text {upper_QoGST }}(r)=\left(G_{m} n-\operatorname{Tr} / G_{b}\right)\left(g-\operatorname{Tr} / G_{b}\right) \text { and } d_{\text {lower_QoGST }}(r)=\left(G_{m} n-m+g-\operatorname{Tr} / G_{b}\right)\left(g-\operatorname{Tr} / G_{b}\right) .(36
$$

\section{Proof: See Appendix V.}

$d_{\text {upper_QoGST }}(r)$ reflects the optimal case with no interference among the groups. In other words, $d_{\text {QoGST }}(r)$ can reach the upper bound $d_{\text {upper_QoGST }}(r)$ when all the groups are orthogonal. However, this condition is satisfied only with the group size $g=1$. In this case, QoGST is reduced to an orthogonal design and obviously we have $d_{\text {QoGST }}(r)=m n(1-\operatorname{Tr} / K)=d_{\text {ortho }}(r)$.

\footnotetext{
${ }^{3}$ In [4], the diversity-multiplexing tradeoff of the orthogonal design with 2 transmit antennas and $n$ receive antennas has been presented as $d(r)=2 n(1-r)$. Through a similar analysis, the tradeoff of the orthogonal design with an arbitrary $m$ and $n$ can be derived as $d(r)=m n(1-\operatorname{Tr} / K)$. We omit the proof due to space limitation.
} 
When there is only one group, i.e., $G=1$, QoGST turns into an $m$-transmit $n$-receive system with a maximum likelihood detector over Rayleigh quasi-static channels. In this case, the upper bound and lower bound converge to $d_{\text {lower_QoGST }}(r)=d_{\text {upper_QoGST }}(r)=(n-r)(m-r)$. Therefore, the diversity-multiplexing tradeoff function of QoGST can be exactly given by $d_{\text {QoGST }}(r)=(n-r)(m-r)$, which is exactly the same as the optimal tradeoff function for an $(m, n)$ over Rayleigh channels as shown in [4].

\section{Tradeoff comparison}

Fig. 4 presents the diversity-multiplexing tradeoff curves of GLST and QoGST when $m=K=4$ and $n=2$. For comparison, we also show the optimal tradeoff curve for an $(m, n)$ over Rayleigh channels. In GLST, assume that there are 2 groups, i.e., $G=2$ and $g_{m}=g_{b}=2$. For QoGST, assume that the group size $g=2$ and thus $G_{m}=G_{b}=2$. Both GLST and QoGST needs $T=2$ time slots. Since $x=T n-K+g_{b}=2$, the lower bound tradeoff function of GLST can be achieved. Therefore, from (34) and (35), the tradeoff function of GLST and the lower and upper bound tradeoff functions of QoGST can be obtained. As Fig. 4 shows, the tradeoff curve of GLST is exactly the same as that of the lower bound of QoGST. This implies that QoGST always has a better diversity-multiplexing tradeoff than GLST. Besides, the upper bound tradeoff of QoGST overlaps with the optimal one. Actually, it is not always the same as the optimal tradeoff. As Fig. 5 shows, when $n$ increases to 3, the maximum multiplexing gain $r_{\max }$ of the optimal one will increase to 3, while $r_{\max }$ of the upper bound of QoGST is only 2. In this case, the lower bound tradeoff of QoGST is even better than the upper bound tradeoff of GLST, thus, indicating a more significant gain.

Fig. 6 and Fig. 7 show the case of $m=K=6$ and $n=4$. Since 6 symbols (antennas) can be divided into 2 groups with 3 symbols (antennas) in each group, or 3 groups with 2 symbols (antennas) in each group, we consider both of these possible partitions. In Fig. 6, the bit stream and transmit antennas are assumed to be separated into 2 groups, i.e., $g_{m}=g_{b}=g=3$ and $G=G_{m}=G_{b}=2$. Recall that in QoGST, STBC is adopted among the groups. Therefore, for a 2-symbol-2-antenna transmission, only $T_{\text {QoGST }}=2$ time slots are needed. However, for GLST, STBC is adopted inside the group. We take the [3,4,3] STBC code given in [23] (pp. 2485, Eqn. (99)), and so $T_{G L S T}=4$. As Fig. 6 shows, QoGST 
can always get a much better diversity-multiplexing tradeoff than GLST as its lower bound is always better than the upper bound tradeoff of GLST. Besides, GLST can only get a maximum multiplexing gain of 1.5, which is lower than that of QoGST. This is because GLST needs more time slots to transmit all $K$ symbols.

Fig. 7 shows the latter partition, i.e., $g_{m}=g_{b}=g=2$ and $G=G_{m}=G_{b}=3$. Here GLST needs 2 time slots, while QoGST needs 4 time slots. From Fig. 7, it can be seen that in this case despite a much higher diversity gain, QoGST has a smaller maximum multiplexing gain due to its lower transmission rate. Nevertheless, if we compare the tradeoff of QoGST with $G_{m}=G_{b}=3$ and $g=2$ to that of GLST with $g_{m}=g_{b}=3$ and $G=2$, it is found that QoGST can always achieve a better diversity gain while keeping the same multiplexing gain as GLST. As Fig. 8 shows, the lower bound tradeoff curve of QoGST is always higher than the upper bound tradeoff curve of GLST. The same conclusion holds true for the comparison of QoGST with $g=3, G_{m}=G_{b}=2$ and GLST with $g_{m}=g_{b}=2, G=3$. Therefore, we can conclude that QoGST always has a better diversity-multiplexing tradeoff than GLST.

\section{Further Results and Discussions}

We have shown that the proposed QoGST has a better diversity-multiplexing tradeoff than GLST. In this section, we further provide the FER performance of both schemes. For the sake of comparison, we also present the FER performance of LSTBC ${ }^{4}$ proposed in [15]. QPSK is assumed to be adopted. As Fig. 9 shows, when $m=4$ and $n=2$, both QoGST and GLST have 2 groups with group size 2. In this case, QoGST can achieve a gain of $3 \mathrm{~dB}$ over GLST at a FER of $10^{-3}$. Besides, in high-SNR conditions, the FER curve of QoGST has a larger slope than that of GLST, which implies that QoGST has a better diversity gain. Notice that these two schemes have the same spectral efficiency. As a result, we can conclude that QoGST achieves a better diversity-multiplexing tradeoff, which is consistent with our analysis in Section IV. Here we did not show the FER curve of LSTBC since it cannot work in this case. Recall that in LSTBC, group detection is performed before space-time decoding. Therefore, the group detector is applied to an $n \times 1$ receive signal vector, which requires that $n>m-g_{m}$. However, for GLST or QoGST, recall that it is only required that $T n>m-g_{m}$ since

\footnotetext{
${ }^{4}$ In order to make a fair comparison, an equal group size is assumed in LSTBC.
} 
group detection is performed after decoding. This implies that both GLST and QoGST have a lower requirement on the number of receive antennas than LSTBC.

When $m=6$ and $n=4$, the transmit antennas of both QoGST and GLST can be divided into 2 or 3 groups. To keep the same transmission rate, the group size of QoGST as well as that of GLST and LSTBC is assumed to be 3 and 2 (or 2 and 3), respectively. As Fig. 9 shows, for the case of $g_{\text {QoGST }}=G_{G L S T}=3$, QoGST can achieve at least $4 \mathrm{~dB}$ gain at a FER of $10^{-3}$, and the FER curve of QoGST is much steeper than that of GLST, which implies a much better diversity gain. Again LSTBC cannot work in this case. When $g_{\text {QoGST }}=G_{G L S T}=2$, QoGST will not achieve such a significant gain as before. Nevertheless, a larger slope and $1 \mathrm{~dB}$ gain can be seen. LSTBC gets the worst performance. At a FER of $10^{-3}, 12 \mathrm{~dB}$ and $11 \mathrm{~dB}$ gains can be achieved by QoGST and GLST, respectively. These observations clearly validate our analysis on the diversity-multiplexing tradeoff.

\section{Conclusions}

To achieve a good tradeoff between multiplexing gain and diversity gain in MIMO systems, this paper proposed two combined STC and LST architectures, which we refer to as QoGST and GLST. In QoGST, the transmit stream is divided into multiple groups and the different groups are encoded together via a quasi-orthogonal inter-group STBC coder. While in GLST, STBC is used inside each group instead of among the groups. We analyzed their diversity-multiplexing tradeoff functions and found that both QoGST and GLST can achieve a good diversity-multiplexing tradeoff. Besides, the lower bound tradeoff of QoGST is always higher than the upper bound tradeoff of GLST, which indicates a significant gain. The simulation results validated our analysis and demonstrated that QoGST can always achieve much better FER performance than GLST and both of them can achieve substantial gains than the existent LSTBC scheme.

\section{Appendix I: Proof of Proposition 2}

For an $[m, T, K]$ STBC $\mathcal{O}_{x}=\left[\mathbf{A}_{1} \mathbf{x}+\mathbf{B}_{1} \mathbf{x}^{*}, \mathbf{A}_{2} \mathbf{x}+\mathbf{B}_{2} \mathbf{x}^{*}, \ldots, \mathbf{A}_{\mathrm{T}} \mathbf{x}+\mathbf{B}_{\mathrm{T}} \mathbf{x}^{*}\right]$, we have

$$
\left(\mathbf{A}_{j}[:, i]\right)^{\prime} \cdot \mathbf{A}_{k}[:, i]=0, \quad\left(\mathbf{B}_{j}[:, i]\right)^{\prime} \cdot \mathbf{B}_{k}[:, i]=0 \text {, and }\left(\mathbf{A}_{j}[:, i]\right)^{\prime} \cdot \mathbf{B}_{k}[:, i]=0
$$


for $j, k=1, \ldots, T, \quad j \neq k, \quad i=1, \ldots, G_{b}$.

Since $\tilde{\mathbf{A}}_{t}^{i}=\mathbf{A}_{t}[:, i] \otimes \mathbf{I}_{g}, \quad \tilde{\mathbf{B}}_{t}^{i}=\mathbf{B}_{t}[:, i] \otimes \mathbf{I}_{g}$, for $i=1, \ldots, G_{b}$ and $t=1, \ldots, T$, then from (37) it can be obtained that

$$
\left(\tilde{\mathbf{A}}_{j}^{i}\right)^{\prime} \cdot \tilde{\mathbf{A}}_{k}^{i}=\mathbf{0}_{g \times g},\left(\tilde{\mathbf{B}}_{j}^{i}\right)^{\prime} \cdot \tilde{\mathbf{B}}_{k}^{i}=\mathbf{0}_{g \times g} \text {, and }\left(\tilde{\mathbf{A}}_{j}^{i}\right)^{\prime} \cdot \tilde{\mathbf{B}}_{k}^{i}=\mathbf{0}_{g \times g}
$$

for $j, k=1, \ldots, T, \quad j \neq k, \quad i=1, \ldots, G_{b}$.

Notice that $\mathbf{b}$ and $\mathbf{b}^{*}$ will not appear in the same time slot $t$. Therefore, by combining (26) and (38), it is obvious that $\mathbf{H}_{t}^{\mathcal{G}_{i}}=\mathbf{H} \tilde{\mathbf{A}}_{t}^{i}+\mathbf{H}^{*} \tilde{\mathbf{B}}_{t}^{i^{*}}, t=1, \ldots, T$, are independent of each other. Furthermore, since the row vectors of $\mathbf{H}_{t}^{\mathcal{G}_{i}}$ are also independent, Proposition 2 can be obtained.

\section{Appendix II: Proof of Theorem 1}

From (5) we know that $\mathbf{Q}_{\mathcal{G}_{i}}^{-1}=\mathbf{H}_{\mathcal{G}_{i}}^{+} \mathbf{P}_{\mathcal{G}_{i}} \mathbf{H}_{\mathcal{G}_{i}}$, where $\mathbf{P}_{\mathcal{G}_{i}}$ is an $N \times N$ projection matrix given by (3). [20] has shown that $\mathbf{P}_{\mathcal{G}_{i}}$ has $N-\left|\overline{\mathcal{G}_{i}}\right|$ unit eigenvalues and $\left|\overline{\mathcal{G}_{i}}\right|$ zero eigenvalues. By applying Singular Value Decomposition (SVD) to $\mathbf{P}_{\mathcal{G}_{i}}$, we have

$$
\mathbf{P}_{\mathcal{G}_{i}}=\mathbf{U}_{\mathcal{G}_{i}}^{+} \Lambda_{\mathcal{G}_{i}} \mathbf{U}_{\mathcal{G}_{i}},
$$

where $\mathbf{U}_{\mathcal{G}_{i}}$ is an $N \times N$ unitary matrix, and $\boldsymbol{\Lambda}_{\mathcal{G}_{i}}$ is an $N \times N$ diagonal matrix whose elements are the descending ordered eigenvalues of $\mathbf{P}_{\mathcal{G}_{i}}$. Therefore, we have

$$
\mathbf{Q}_{\mathcal{G}_{i}}^{-1}=\mathbf{H}_{\mathcal{G}_{i}}^{+} \mathbf{P}_{\mathcal{G}_{i}} \mathbf{H}_{\mathcal{G}_{i}}=\mathbf{V}_{\mathcal{G}_{i}}^{+} \boldsymbol{\Lambda}_{\mathcal{G}_{i}} \mathbf{V}_{\mathcal{G}_{i}}=\sum_{i=1}^{N-m+\left|\mathcal{G}_{i}\right|} \mathbf{v}_{i}^{+} \mathbf{v}_{i},
$$

where $\mathbf{V}_{\mathcal{G}_{i}}=\mathbf{U}_{\mathcal{G}_{i}} \mathbf{H}_{\mathcal{G}_{i}}$ with the row vector $\mathbf{v}_{i}, i=1, \ldots, N$.

Notice that $\mathbf{P}_{\mathcal{G}_{i}}$ is independent of $\mathbf{H}_{\mathcal{G}_{i}}$ and the row vectors of $\mathbf{H}_{\mathcal{G}_{i}}$ are independent Gaussian distributed. Then, from [21] (pp. 91, Lemma 7.2) we know that $\mathbf{Q}_{\mathcal{G}_{i}}^{-1}$ is Wishart distributed with $N-m+\left|\mathcal{G}_{i}\right|$ degrees of freedom. Therefore, the joint pdf of $\left\{\lambda_{j}\right\}_{j=1, \ldots, \mathcal{G}_{i} \mid}$ is given by (31) [22]. 


\section{Appendix III: Proof of Theorem 2}

From [4], we know that the outage probability of the whole channel is

$$
P_{\text {out }}(R) \doteq P[I(\mathbf{b} ; \mathbf{y} \mid \mathbf{H}=H)<R] .
$$

Clearly, with GZF any group in outage will lead to the outage event of the whole system. Therefore, we have

$$
P_{\text {out }}(R)=1-\prod_{i=1}^{G}\left(1-P_{\mathcal{G}_{i}, \text { out }}\left(R_{\mathcal{G}_{i}}\right)\right)
$$

Furthermore, since $P_{\mathcal{G}_{i}, \text { out }}(R) \doteq S N R^{-d_{\mathcal{G}_{i} \text {,out }}\left(r_{\mathcal{G}_{i}}\right)}, \quad P_{\text {out }}(R) \doteq S N R^{-d_{\text {out }}(r)}$, where $r_{\mathcal{G}_{i}}=R_{\mathcal{G}_{i}} / \log (S N R)$ is the spatial multiplexing gain of group $\mathcal{G}_{i}$ and $r=\sum_{i=1}^{G} r_{\mathcal{G}_{i}}$, we obtain from (42) that

$$
d_{\text {out }}(r)=\left\{\min _{i=1, \ldots, G}\left\{d_{\mathcal{G}_{i}, \text { out }}\left(r_{\mathcal{G}_{i}}\right)\right\} ; \sum_{i=1}^{G} r_{\mathcal{G}_{i}}\right\} .
$$

Similar to [4], for a sufficiently long block length $L \geq N-\left|\overline{\mathcal{G}}_{x}\right|+\left|\mathcal{G}_{x}\right|-1$ (where $\mathcal{G}_{x}=\arg \max _{i=1, \ldots, G}\left|\mathcal{G}_{i}\right|$ ), and by assuming an input to be i.i.d. Gaussian random code, we can obtain the overall system tradeoff function as

$$
d(r)=d_{\text {out }}(r)=\left\{\min _{i=1, \ldots, G}\left\{d_{\mathcal{G}_{i}, \text { out }}\left(r_{\mathcal{G}_{i}}\right)\right\} ; \sum_{i=1}^{G} r_{\mathcal{G}_{i}}\right\} .
$$

\section{Appendix IV: Proof of Theorem 3}

We begin by considering the upper bound. From (3) and (5), we know that $\mathbf{Q}_{\mathcal{G}_{i}}^{-1}=\mathbf{H}_{\mathcal{G}_{i}}^{+} \mathbf{P}_{\mathcal{G}_{i}} \mathbf{H}_{\mathcal{G}_{i}}=\mathbf{H}_{\mathcal{G}_{i}}^{+}\left(\mathbf{I}_{N}-\mathbf{H}_{\overline{\mathcal{G}}_{i}}\left(\mathbf{H}_{\overline{\mathcal{G}}_{i}}^{+} \mathbf{H}_{\overline{\mathcal{G}}_{i}}\right)^{-1} \mathbf{H}_{\overline{\mathcal{G}}_{i}}^{+}\right) \mathbf{H}_{\mathcal{G}_{i}}=\mathbf{H}_{\mathcal{G}_{i}}^{+} \mathbf{H}_{\mathcal{G}_{i}}-\boldsymbol{\Phi}_{\mathcal{G}_{i}}$, where $\boldsymbol{\Phi}_{\mathcal{G}_{i}}=\mathbf{H}_{\mathcal{G}_{i}}^{+} \mathbf{H}_{\overline{\mathcal{G}}_{i}}\left(\mathbf{H}_{\overline{\mathcal{G}}_{i}}^{+} \mathbf{H}_{\overline{\mathcal{G}}_{i}}\right)^{-1} \mathbf{H}_{\overline{\mathcal{G}}_{i}}^{+} \mathbf{H}_{\mathcal{G}_{i}}$.

Obviously, $\boldsymbol{\Phi}_{\mathcal{G}_{i}}$ is positive-definite Hermitian, i. e., $\quad \boldsymbol{\Phi}_{\mathcal{G}_{i}}=\mathbf{H}_{\mathcal{G}_{i}}^{+} \mathbf{H}_{\mathcal{G}_{i}}-\mathbf{Q}_{\mathcal{G}_{i}}^{-1} \geq \mathbf{0}$ (only when $\left|\overline{\mathcal{G}_{i}}\right|=0$, i.e., $\left.G=1, \quad \boldsymbol{\Phi}_{\mathcal{G}_{i}}=\mathbf{0}\right)$. Therefore, we have $\operatorname{det}\left(\mathbf{Q}_{\mathcal{G}_{i}}^{-1}\right) \leq \operatorname{det}\left(\mathbf{H}_{\mathcal{G}_{i}}^{+} \mathbf{H}_{\mathcal{G}_{i}}\right)$ and for an arbitrary R,

$$
P\left(\operatorname{det}\left(\mathbf{Q}_{\mathcal{G}_{i}}^{-1}\right)<R\right) \geq P\left(\operatorname{det}\left(\mathbf{H}_{\mathcal{G}_{i}}^{+} \mathbf{H}_{\mathcal{G}_{i}}\right)<R\right) .
$$

As shown in Section IV. A, the outage probability of any group $\mathcal{G}_{i}$ is given by

$$
P_{\mathcal{G}_{i} \text { out }}\left(R_{\mathcal{G}_{i}}\right) \doteq P\left[\log \operatorname{det}\left(\mathbf{I}_{\left|\mathcal{G}_{i}\right|}+S N R \cdot \mathbf{Q}_{\mathcal{G}_{i}}^{-1}\right)<R_{\mathcal{G}_{i}}\right] \approx P\left[\operatorname{det}\left(\mathbf{Q}_{\mathcal{G}_{i}}^{-1}\right)<S N R^{-\left(g_{b}-r_{\mathcal{G}_{i}}\right)}\right] .
$$

By combining (44) and (45), it can be obtained that 


$$
P_{\mathcal{G}_{i} \text {,out }}\left(R_{\mathcal{G}_{i}}\right) \geq P\left(\operatorname{det}\left(\mathbf{H}_{\mathcal{G}_{i}}^{+} \mathbf{H}_{\mathcal{G}_{i}}\right)<S N R^{-\left(g_{b}-r_{\mathcal{G}_{i}}\right)}\right)
$$

From Proposition 1 in Section III A.2, we know that $\operatorname{det}\left(\mathbf{H}_{\mathcal{G}_{i}^{+}}^{+} \mathbf{H}_{\mathcal{G}_{i}}\right)=\left(\sum_{l=1}^{g_{m}} \mathbf{h}_{i, l}^{+} \mathbf{h}_{i, l}\right)^{g_{b}}$. Let $x=\sum_{l=1}^{g_{m}} \mathbf{h}_{i, l}^{+} \mathbf{h}_{i, l}$. Obviously, $x$ is chi-square distributed with dimension $g_{m} n$. Therefore,

$$
P\left(\operatorname{det}\left(\mathbf{H}_{\mathcal{G}_{i}}^{+} \mathbf{H}_{\mathcal{G}_{i}}\right)<S N R^{-\left(g_{b}-r_{\mathcal{G}_{i}}\right)}\right)=P\left(x<S N R^{-\left(1-r_{\mathcal{G}_{i}} / g_{b}\right)}\right)=S N R^{-g_{m} n\left(1-r_{\mathcal{G}_{i}} / g_{b}\right)} .
$$

Let $P\left(\operatorname{det}\left(\mathbf{H}_{\mathcal{G}_{i}}^{+} \mathbf{H}_{\mathcal{G}_{i}}\right)<S N R^{-\left(g_{b}-r_{\mathcal{G}_{i}}\right)}\right) \doteq S N R^{-d^{*}\left(r_{\mathcal{G}_{i}}\right)}$. From (46), we get

$$
d_{\mathcal{G}_{i}, \text { out }}\left(r_{\mathcal{G}_{i}}\right) \leq d^{*}\left(r_{\mathcal{G}_{i}}\right)=g_{m} n\left(1-r_{\mathcal{G}_{i}} / g_{b}\right)
$$

Assume that equal rate allocation scheme is applied. By applying Theorem 2 we have

$$
d(r) \leq d^{*}(r)=g_{m} n(1-r / K)
$$

As stated in Section IV, GLST should have the same diversity gain as an $m$-transmit $T n$-receive system over a channel given by (14) with a GZF. However, in contrast to an $(m, T n)$ system in which all the $K$ symbols are transmitted simultaneously, $T$ transmit time slots are needed in GLST. Therefore, despite the same diversity gain, the multiplexing gain of GLST should be $1 / T$ of that of this $(m, T n)$ system. By substituting $\operatorname{Tr}$ for $r$ in (49), we have

$$
d_{G L S T}(r) \leq d_{\text {upper_GLST }}(r)=g_{m} n(1-\operatorname{Tr} / K) .
$$

The upper bound can be achieved when $\boldsymbol{\Phi}_{\mathcal{G}_{i}}=\mathbf{H}_{\mathcal{G}_{i}}^{+} \mathbf{H}_{\mathcal{G}_{i}}-\mathbf{Q}_{\mathcal{G}_{i}}^{-1}=\mathbf{0}$, i.e., $G=1$.

Now consider the lower bound. From (14) it can be seen that $\mathbf{H}_{\mathcal{G}_{i}}$ is independent to $\mathbf{H}_{\mathcal{G}_{j}}$, for any $i \neq j$. Therefore, $\mathbf{P}_{\mathcal{G}_{i}}$ is independent of $\mathbf{H}_{\mathcal{G}_{i}}$ and from (40) we have

$$
\mathbf{Q}_{\mathcal{G}_{i}}^{-1}=\sum_{i=1}^{T n-K+g_{b}} \mathbf{v}_{i}^{+} \mathbf{v}_{i}
$$

Assume that $\mathbf{H}_{\mathcal{G}_{i}}^{+}=\left[\tilde{\mathbf{h}}_{1}^{+}, \tilde{\mathbf{h}}_{2}^{+}, \ldots, \tilde{\mathbf{h}}_{T n}^{+}\right]$, where $\tilde{\mathbf{h}}_{i}$ is the $i^{\text {th }}$ row vector of $\mathbf{H}_{\mathcal{G}_{i}}, i=1, \ldots, T n$. Obviously, only $\left\{\tilde{\mathbf{h}}_{i}\right\}_{i=1, \ldots, j}$ are independent where $j \leq n$. Therefore, let $x=\min \left(n, T n-K+g_{b}\right)$ and $\tilde{\mathbf{Q}}_{\mathcal{G}_{i}}^{-1}=\sum_{i=1}^{x} \mathbf{v}_{i}^{+} \mathbf{v}_{i}$. Then for an arbitrary $R$, clearly we have

$$
P\left(\operatorname{det}\left(\mathbf{Q}_{\mathcal{G}_{i}}^{-1}\right)<R\right) \leq P\left(\operatorname{det}\left(\tilde{\mathbf{Q}}_{\mathcal{G}_{i}}^{-1}\right)<R\right)
$$

The outage probability of any group $\mathcal{G}_{i}$ of GLST is thus upper bounded by 


$$
P_{\mathcal{G}_{i}, \text { out }}\left(R_{\mathcal{G}_{i}}\right) \leq P_{\mathcal{G}_{i} \text {,out }}^{*}\left(R_{\mathcal{G}_{i}}\right) \doteq P\left[\log \operatorname{det}\left(\mathbf{I}_{\left|\mathcal{G}_{i}\right|}+S N R \cdot \tilde{\mathbf{Q}}_{\mathcal{G}_{i}}^{-1}\right)<R_{\mathcal{G}_{i}}\right]
$$

Considering that $\tilde{\mathbf{Q}}_{\mathcal{G}_{i}}^{-1}$ is Wishart distributed with $x$ degrees of freedom, from Theorem 2 its corresponding tradeoff function can be obtained as

$$
d^{*}\left(r_{\mathcal{G}_{i}}\right)=\left(x-r_{\mathcal{G}_{i}}\right)\left(g_{b}-r_{\mathcal{G}_{i}}\right)
$$

Likewise, by assuming equal rate allocation and substituting $\operatorname{Tr}$ for $r$, we have

$$
d_{G L S T}(r) \geq d_{\text {lower_GLST }}(r)=(x-\operatorname{Tr} / G)\left(g_{b}-T r / G\right),
$$

where $x=\min \left(n, T n-K+g_{b}\right)$.

The lower bound can be achieved when $x=T n-K+g_{b}$.

By combining (50) and (55), (35) can be obtained.

\section{Appendix V: Proof of Theorem 4}

We begin by considering the upper bound. Similar to Appendix IV, for any group $\mathcal{G}_{i}$,

$$
\mathbf{Q}_{\mathcal{G}_{i}}^{-1}=\mathbf{H}_{\mathcal{G}_{i}}^{+} \mathbf{H}_{\mathcal{G}_{i}}-\mathbf{\Phi}_{\mathcal{G}_{i}} \leq \mathbf{H}_{\mathcal{G}_{i}}^{+} \mathbf{H}_{\mathcal{G}_{i}}
$$

where $\boldsymbol{\Phi}_{\mathcal{G}_{i}}=\mathbf{H}_{\mathcal{G}_{i}}^{+} \mathbf{H}_{\overline{\mathcal{G}_{i}}}\left(\mathbf{H}_{\overline{\mathcal{G}}_{i}}^{+} \mathbf{H}_{\overline{\mathcal{G}_{i}}}\right)^{-1} \mathbf{H}_{\overline{\mathcal{G}}_{i}}^{+} \mathbf{H}_{\mathcal{G}_{i}}$. It turns out that $\boldsymbol{\Phi}_{\mathcal{G}_{i}}=\mathbf{0}$ only when $g=1$. In this case, QoGST is reduced to the orthogonal design and no interference exists among the groups. When $g>1$, our scheme cannot eliminate the inter-group interference entirely. $\mathbf{H}_{\mathcal{G}_{i}}$ will be dependent to $\mathbf{H}_{\overline{\mathcal{G}_{i}}}$, but not orthogonal to $\mathbf{H}_{\overline{\bar{q}_{i}}}$.

From Proposition 2 in Section III B.2, we know that $\mathbf{X}=\mathbf{H}_{\mathcal{G}_{i}}^{+} \mathbf{H}_{\mathcal{G}_{i}}$ should be Wishart distributed with $G_{m} n$ degrees of freedom, which corresponds to a tradeoff function $d^{*}(r)=\left(G_{m} n-r\right)(g-r)$. Therefore, by applying a similar proof shown in Appendix IV, we can get

$$
d_{\mathcal{G}_{i} \text {,out }}\left(r_{\mathcal{G}_{i}}\right) \leq d^{*}\left(r_{\mathcal{G}_{i}}\right)=\left(G_{m} n-r_{\mathcal{G}_{i}}\right)\left(g-r_{\mathcal{G}_{i}}\right) .
$$

Assume equal rate in each group. Then, by substituting $\operatorname{Tr} / G_{b}$ for $r_{\mathcal{G}_{i}}$ into (57), it can be obtained that

$$
d_{\text {QoGST }}(r) \leq d_{\text {upper_QoGST }}(r)=\left(G_{m} n-\operatorname{Tr} / G_{b}\right)\left(g-\operatorname{Tr} / G_{b}\right) .
$$

The upper bound can be achieved when $\boldsymbol{\Phi}_{\mathcal{G}_{i}}=\mathbf{H}_{\mathcal{G}_{i}}^{+} \mathbf{H}_{\mathcal{G}_{i}}-\mathbf{Q}_{\mathcal{G}_{i}}^{-1}=\mathbf{0}$, i.e., $g=1$. 
Now, consider a $G_{m} n \times K$ Rayleigh channel $\tilde{\mathbf{H}}$, whose entries are all independent Gaussian random variables with mean zero and unit variance. Next, let us separate it into $G_{b}$ groups; namely, $\tilde{\mathbf{H}}=\left[\tilde{\mathbf{H}}_{\mathcal{G}_{1}}, \ldots, \tilde{\mathbf{H}}_{\mathcal{G}_{G_{b}}}\right]$, where $\tilde{\mathbf{H}}_{\mathcal{G}_{i}}$ is $G_{m} n \times g$, for $i=1, \ldots, G_{b}$. Then, we have

$$
\tilde{\mathbf{Q}}_{\mathcal{G}_{i}}^{-1}=\tilde{\mathbf{H}}_{\mathcal{G}_{i}}^{+} \tilde{\mathbf{H}}_{\mathcal{G}_{i}}-\tilde{\mathbf{H}}_{\mathcal{G}_{i}}^{+} \tilde{\mathbf{H}}_{\overline{\mathcal{G}_{i}}}\left(\tilde{\mathbf{H}}_{\overline{\mathcal{G}}_{i}}^{+} \tilde{\mathbf{H}}_{\overline{\mathcal{G}_{i}}}\right)^{-1} \tilde{\mathbf{H}}_{\overline{\mathcal{G}}_{i}}^{+} \tilde{\mathbf{H}}_{\mathcal{G}_{i}}
$$

By comparing to (56), it can be seen that $\mathbf{H}_{\mathcal{G}_{i}}^{+} \mathbf{H}_{\mathcal{G}_{i}}$ and $\tilde{\mathbf{H}}_{\mathcal{G}_{i}}^{+} \tilde{\mathbf{H}}_{\mathcal{G}_{i}}$ have the same distribution. Since $\mathbf{H}_{\mathcal{G}_{i}}$ is quasi-orthogonal to $\mathbf{H}_{\overline{\mathcal{G}_{i}}}$ but $\tilde{\mathbf{H}}_{\mathcal{G}_{i}}$ is independent to $\tilde{\mathbf{H}}_{\overline{\mathcal{G}_{i}}}$, it can be expected that $\operatorname{det}\left(\mathbf{Q}_{\mathcal{G}_{i}}^{-1}\right)$ should have a better cumulative density function (cdf) than $\operatorname{det}\left(\tilde{\mathbf{Q}}_{\mathcal{G}_{i}}^{-1}\right)$. We resort to Monte Carlo simulations to verify this conclusion. Fig. 3 shows the pdf and $\operatorname{cdf}$ of $X_{1}=\operatorname{det}\left(\mathbf{Q}_{\mathcal{G}_{i}}^{-1}\right)$ and $X_{2}=\operatorname{det}\left(\tilde{\mathbf{Q}}_{\mathcal{G}_{i}}^{-1}\right)$ for $K=4, n=2$ and $G_{m}=g=2$. It can be seen that for an arbitrary $R$, we always have

$$
P\left(X_{1}<R\right) \leq P\left(X_{2}<R\right)
$$

Actually, the conclusion is the same for any other values. Due to limited space, however, no further results are given.

Next, from (60), we have

$$
P_{\mathcal{G}_{i}, \text { out }}^{\text {QoST }}\left(R_{\mathcal{G}_{i}}\right) \doteq P\left[\operatorname{det}\left(\mathbf{Q}_{\mathcal{G}_{i}}^{-1}\right)<S N R^{-\left(g-r_{\mathcal{G}_{i}}\right)}\right] \leq P_{\mathcal{G}_{i}, \text { out }}^{\left(K, G_{m} n\right) G Z F}\left(R_{\mathcal{G}_{i}}\right) \doteq P\left(\operatorname{det}\left(\tilde{\mathbf{Q}}_{\mathcal{G}_{i}}^{-1}\right)<S N R^{-\left(g-r_{\mathcal{G}_{i}}\right)}\right)
$$

We have proved that for a $\left(K, G_{m} n\right)$ GZF, when equal rate allocation is adopted, the diversity-multiplexing tradeoff function of group $\mathcal{G}_{i}$ is given by

$$
d_{\mathcal{G}_{i}, \text { out }}^{\left(K, G_{m} n\right) G Z F}\left(r_{\mathcal{G}_{i}}\right)=\left(G_{m} n-m+g-r_{\mathcal{G}_{i}}\right)\left(g-r_{\mathcal{G}_{i}}\right)
$$

Therefore,

$$
d_{\mathcal{G}_{i}, \text { out }}^{\text {QoGST }}\left(r_{\mathcal{G}_{i}}\right) \geq d_{\mathcal{G}_{i}, \text { out }}^{\left(K, G_{m} n\right) G Z F}\left(r_{\mathcal{G}_{i}}\right)=\left(G_{m} n-m+g-r_{\mathcal{G}_{i}}\right)\left(g-r_{\mathcal{G}_{i}}\right)
$$

Similarly, by substituting $\operatorname{Tr} / G_{b}$ for $r_{\mathcal{G}_{i}}$ into (63), we get

$$
d_{\text {QoGST }}(r) \geq d_{\text {lower_QoGST }}(r)=\left(G_{m} n-m+g-\operatorname{Tr} / G_{b}\right)\left(g-\operatorname{Tr} / G_{b}\right) .
$$

By combining (58) and (64), (36) can be obtained.

\section{REFERENCES}

[1] R. D. Murch and K. B. Letaief, “Antenna systems for broadband wireless access,” IEEE Communications 
Magazine, Vol. 40, No. 4, pp. 76-83, April 2002.

[2] J. H. Winters, “The diversity gain of transmit diversity in wireless systems with Rayleigh fading," IEEE Trans. Veh. Technol., vol. 47, no. 1, pp. 119-123, Feb. 1998.

[3] G. J. Foschini and M. J. Gans, “On limits of wireless communications in a fading environment when using multiple antennas,” Wireless Personal Commun., vol. 6, pp. 311-335, March 1998.

[4] L. Zheng and D. N. C. Tse, "Diversity and multiplexing: a fundamental tradeoff in multiple-antenna channels,” IEEE Trans. Inf. Theory, vol. 49, no. 5, pp. 1073-1096, May 2003.

[5] S. M. Alamouti, “A simple transmit diversity technique for wireless communications,” IEEE J. Select Areas in Commun., vol. 16, no. 8, pp. 1451-1458, Oct. 1998.

[6] V. Tarokh, H. Jafarkhani and A. R. Calderbank, “Space-time block codes from orthogonal designs,” IEEE Trans. Inf. Theory, vol. 45, no. 5, pp. 1456-1467, July. 1999.

[7] V. Tarokh, N. Seshadri and A. R. Calderbank, "Space-time codes for high data rate wireless communication: performance criterion and code construction,” IEEE Trans. Inf. Theory, vol. 44, no. 2, pp. 744-765, Mar. 1998.

[8] P. W. Wolniansky, G. J. Foschini, G. D. Golden and R. A. Valenzuela, "V-BLAST: an architecture for realizing very high data rates over the rich-scattering wireless channel,” in Proc. ISSSE'98, Pisa, Italy, pp. 295-300, Sep. 1998.

[9] G. J. Foschini, "Layered space-time architecture for wireless communication in a fading environment when using multi-element antennas,” Bell Labs Tech. J., vol. 1, no. 2, pp. 41-59, 1996.

[10] H. Yao and G. W. Wornell, "Achieving the full MIMO diversity-multiplexing frontier with rotation-based space-time codes," in Proc. Allerton Conf. Commun., Control and Computing, Illinois, Oct. 2003.

[11] H. El Gamal, G. Caire and M. O. Damen, "Lattice coding and decoding achieve the optimal diversity-multiplexing tradeoff of MIMO channels,” IEEE Trans. Inf. Theory, vol. 50, pp. 968-985, June 2004.

[12] S. Tavildar and P. Viswanath, "Permutation codes for parallel fading channel: achieving the diversity-multiplexing tradeoff,” in Proc. ISIT’04, Illinois, June, 2004.

[13] X. Ma, and G. B. Giannakis, "Complex Field Coded MIMO Systems: Performance, Rate, and Tradeoffs,” Wireless Communications and Mobile Computing, pp. 693-717, Nov. 2002.

[14] V. Tarokh, A. Naguib, N. Seshadri and A. R. Calderbank, "Combined array processing and space-time coding,” IEEE Trans. Inf. Theory, vol. 45, no. 4, pp. 1121-1128, May 1999.

[15] N. Prasad and M. K. Varanasi, “Optimum efficiently decodable layered space-time block codes,” in Proc. the $35^{\text {th }}$ Asilomar Conference on Signals, Systems and Computers, pp. 227-231, Nov. 2001.

[16] I. Kim and V. Tarokh, "Variable-rate space-time block codes in M-ary PSK systems," IEEE J. Select Areas in Commun., vol. 21, no. 3, pp. 362-373, Apr. 2003.

[17] M. Tao and R. S. Cheng, "Generalized layered space-time codes for high data rate wireless communications,” IEEE Trans. Wireless Commun., vol. 3, pp. 1067-1075, July 2004.

[18] H. Jafarkhani, “A quasi-orthogonal space-time block code,” IEEE Trans. Commun., vol. 49, no. 1, pp. 1-4, Jan. 2001.

[19] B. Hassibi and B. M. Hochwald, "High-rate codes that are linear in space and time," IEEE Trans. Inf. Theory, vol. 48, no. 7, pp. 1804-1824, July 2002.

[20] E. A. Fain and M. K. Varanasi, "Diversity order gain for narrow-band multiuser communications with pre-combining group detection,” IEEE Trans. Commun., vol. 48, no. 4, pp. 533-536, Apr. 2000.

[21] M. Bilodeau and D. Brenner, Theory of Multivariate Statistics: Springer-Verlag, 1999.

[22] R. J. Muirhead, Aspects of Multivariate Statistical Theory: John Wiley and Sons, 1982.

[23] X. Liang, “Orthogonal designs with maximum rates,” IEEE Trans. Inf. Theory, vol. 49, no. 10, pp. 2468-2503, Oct. 2003. 

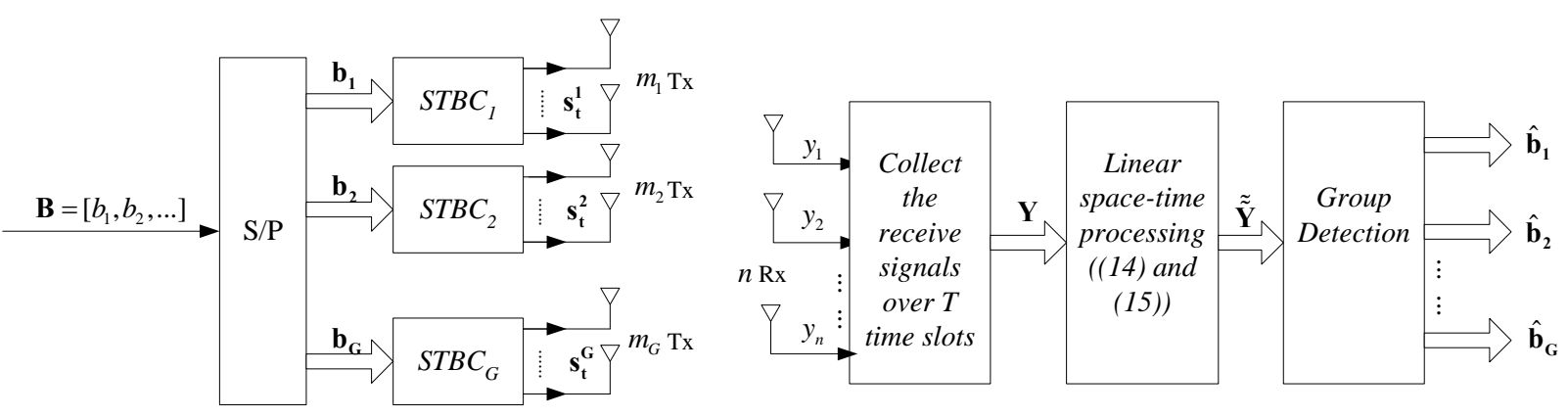

Fig. 1: Block diagram of GLST
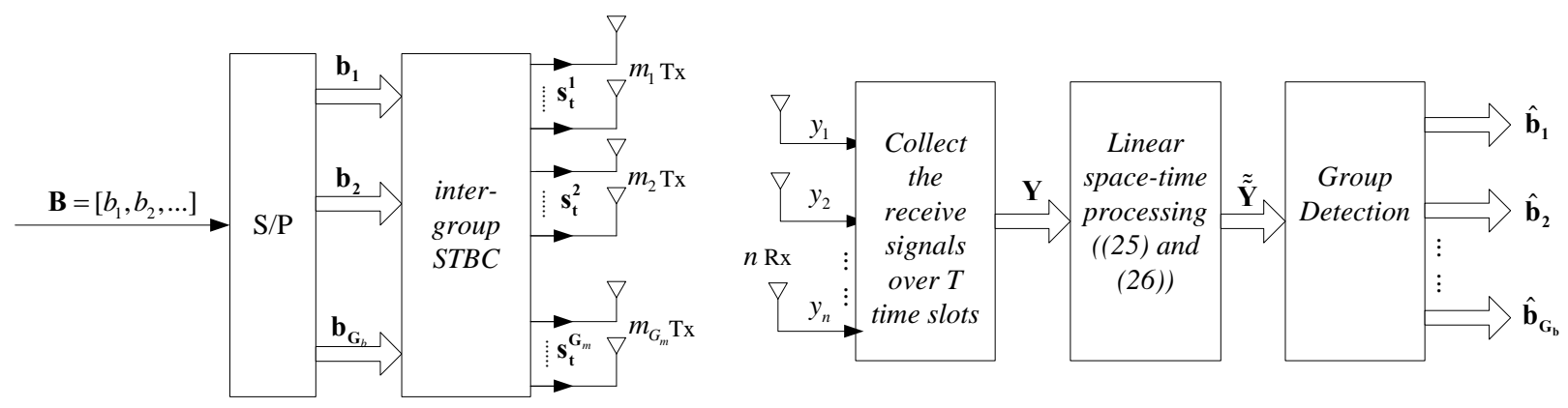

Fig. 2: Block diagram of QoGST 


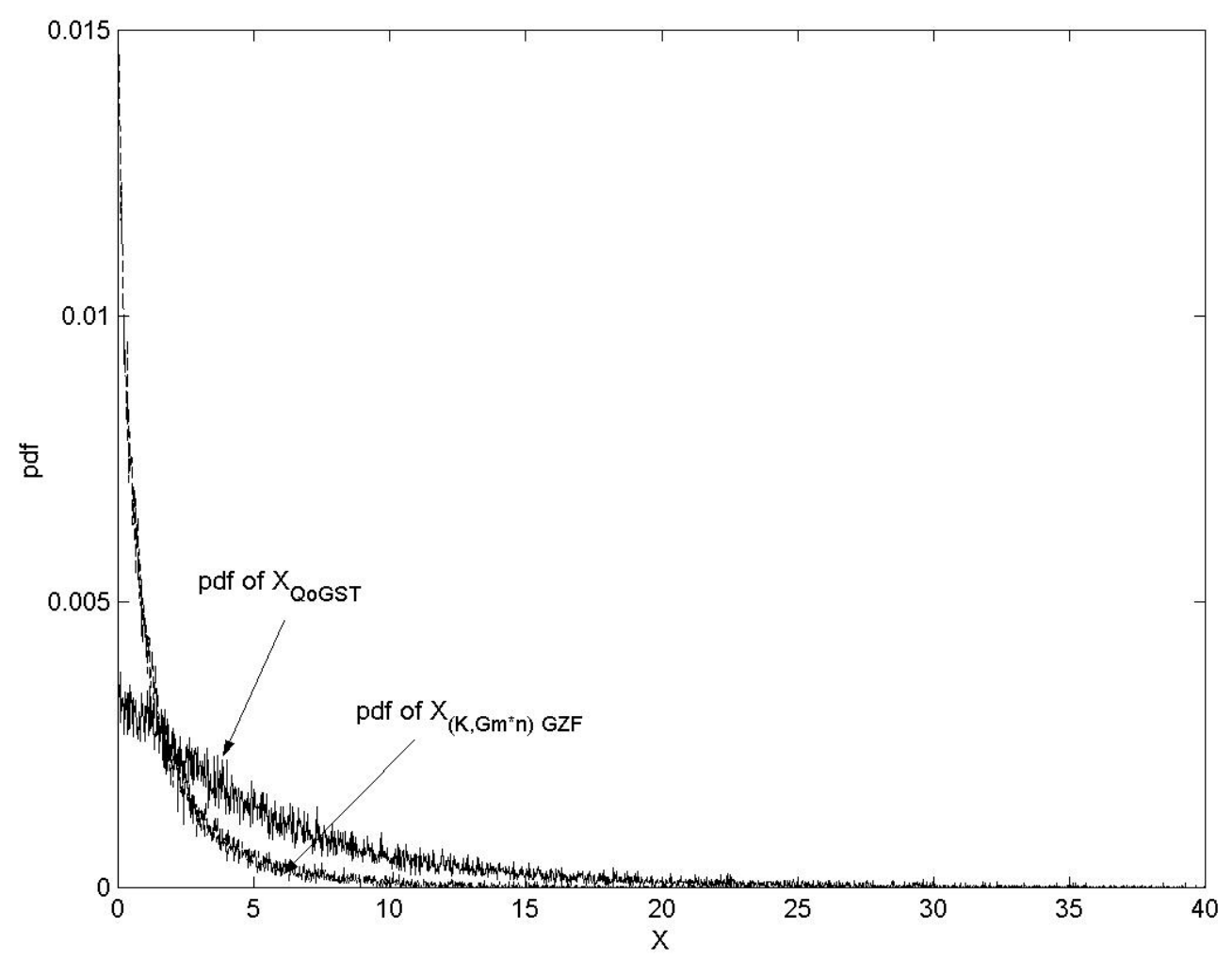

Fig. 3(a): pdf of $X_{1}^{\text {QoGST }}=\operatorname{det}\left(\mathbf{Q}_{\mathcal{G}_{i}}^{-1}\right)$ and $X_{2}^{\left(K, G_{m} n\right) G Z F}=\operatorname{det}\left(\tilde{\mathbf{Q}}_{\mathcal{G}_{i}}^{-1}\right)$ when $K=4, n=2, \quad G_{m}=2$ and $g=2$.

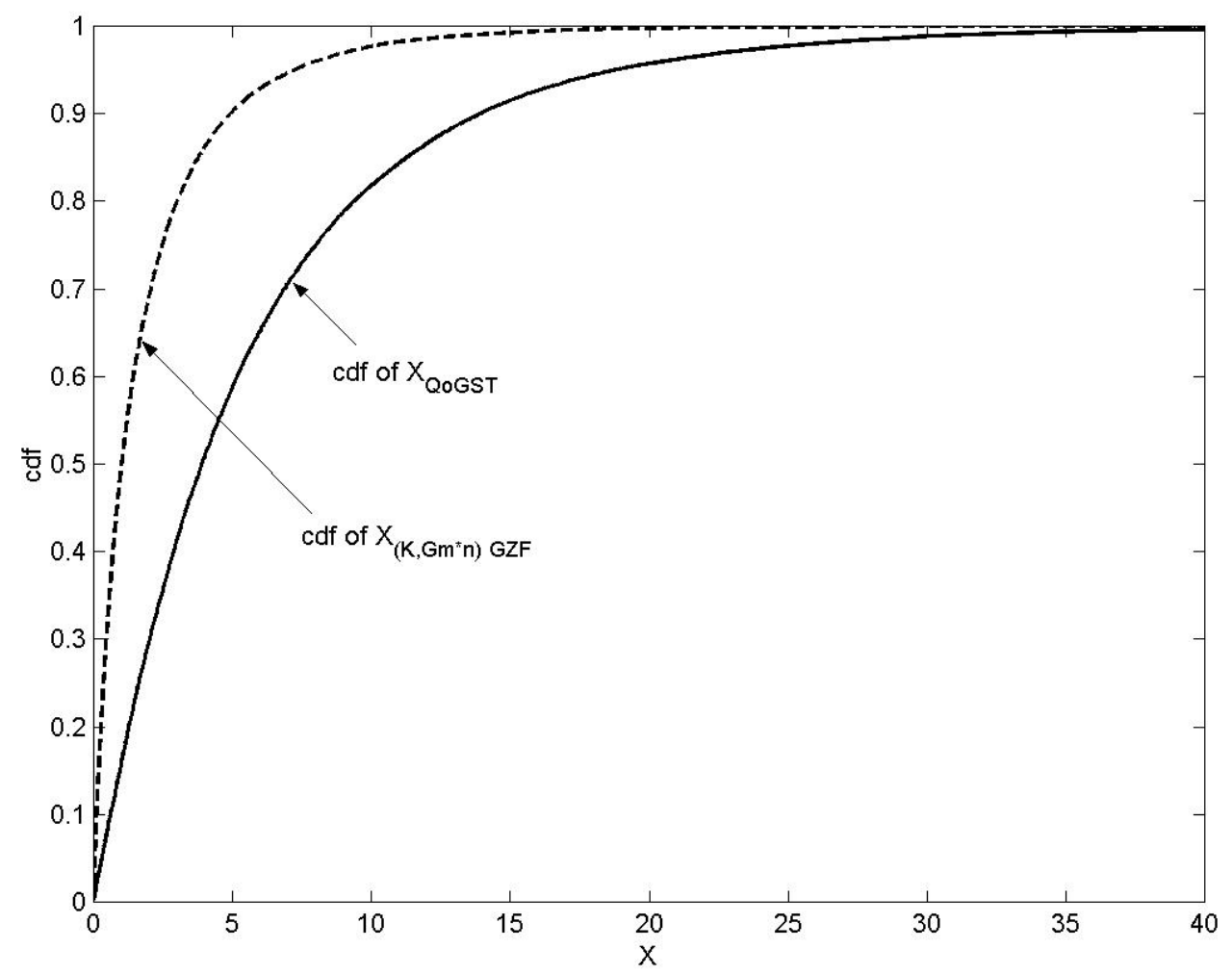

Fig. 3(b): $\quad$ cdf of $X_{1}^{\text {QoGST }}=\operatorname{det}\left(\mathbf{Q}_{\mathcal{G}_{i}}^{-1}\right)$ and $X_{2}^{\left(K, G_{m} n\right) G Z F}=\operatorname{det}\left(\tilde{\mathbf{Q}}_{\mathcal{G}_{i}}^{-1}\right)$ when $K=4, n=2, G_{m}=2$ and $g=2$. 


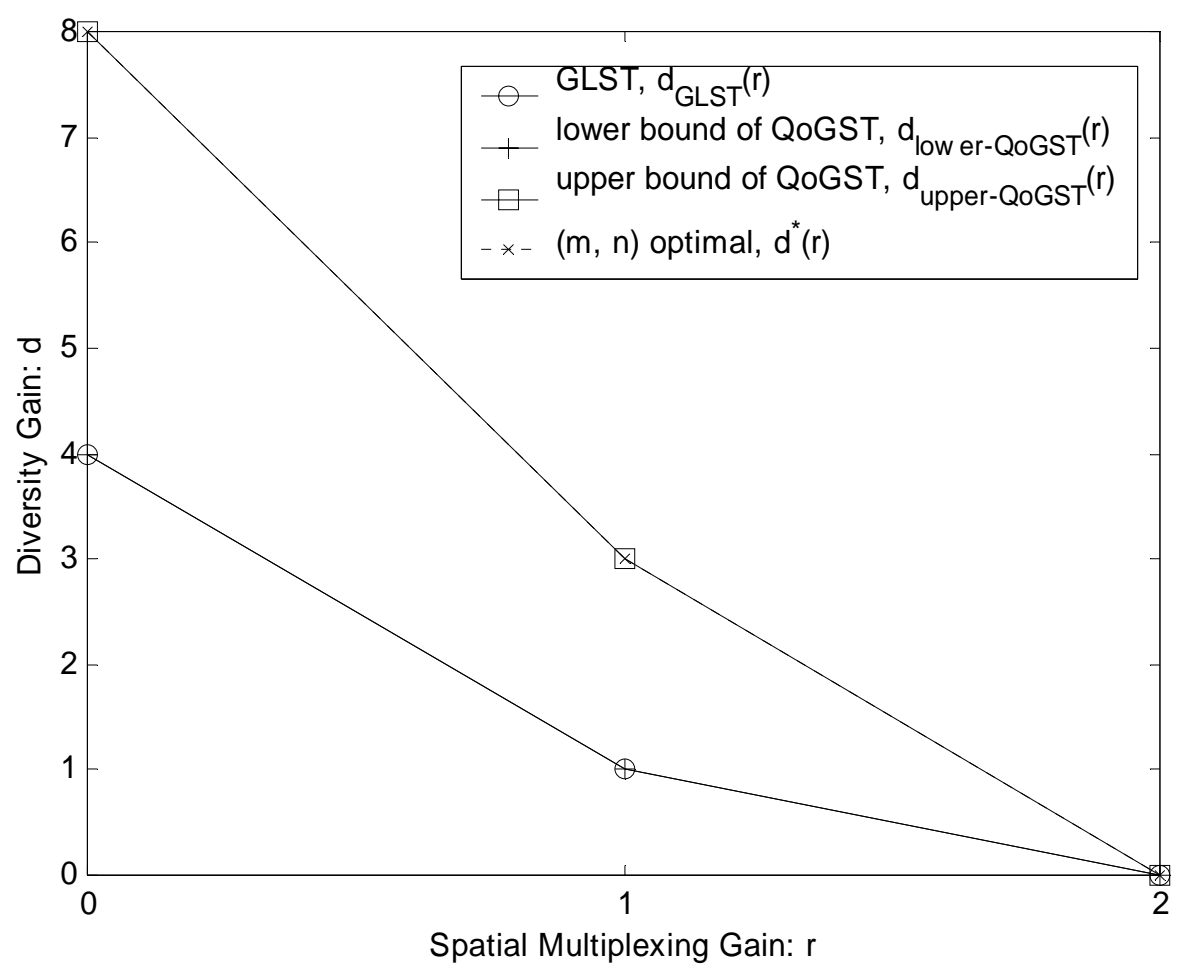

Fig. 4: Diversity-multiplexing tradeoff curves of GLST, the upper and lower bounds of QoGST and the optimal one when $m=K=4, n=2$. For GLST, $G=2, g_{m}=g_{b}=2$, and $T=2$. For QoGST, $g=2, G_{m}=G_{b}=2$, and $T=2$.

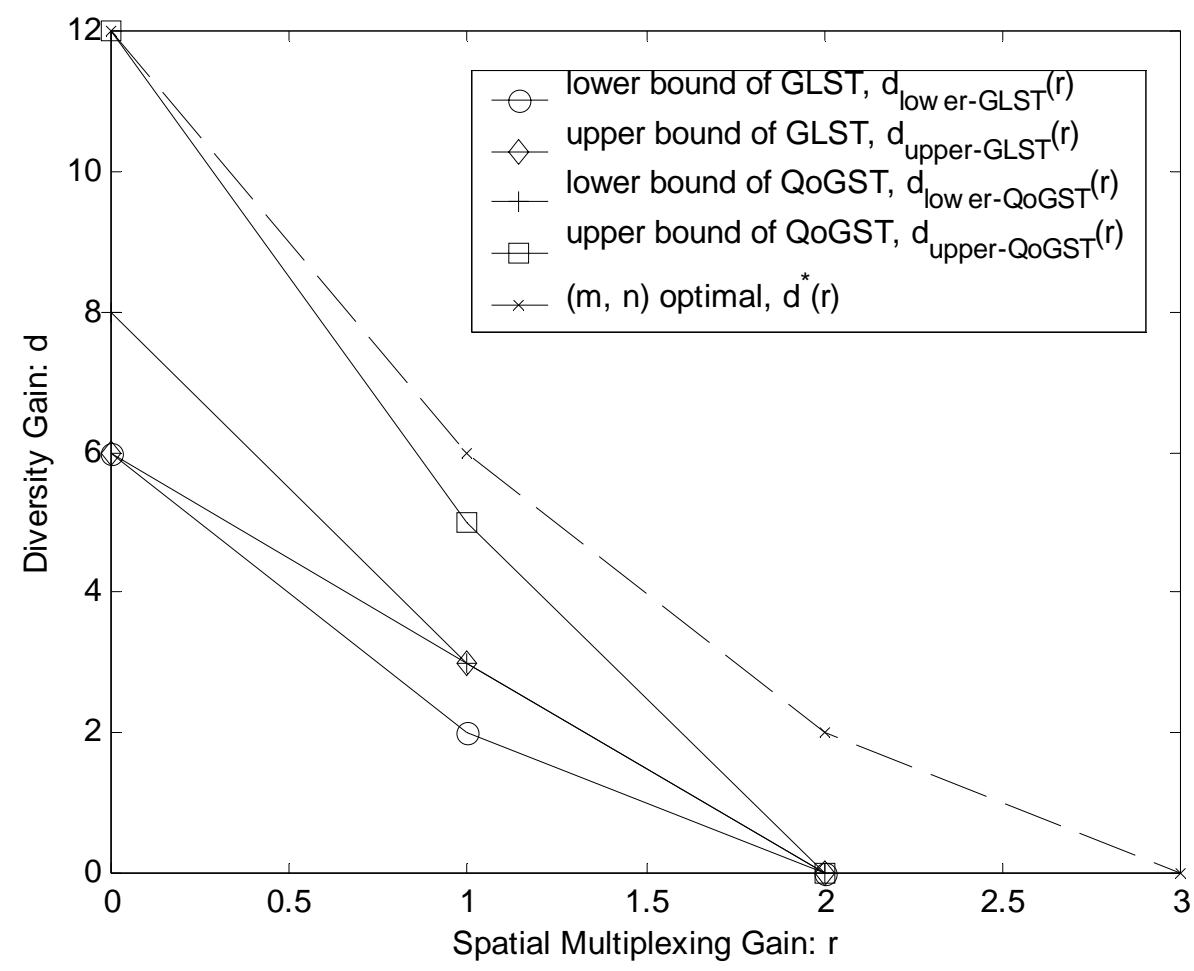

Fig. 5: Diversity-multiplexing tradeoff curves of the upper and lower bounds of GLST, the upper and lower bounds of QoGST and the optimal one when $m=K=4, n=3$. For GLST, $G=2, g_{m}=g_{b}=2, T=2$. For QoGST, $g=2$, $G_{m}=G_{b}=2, T=2$. 


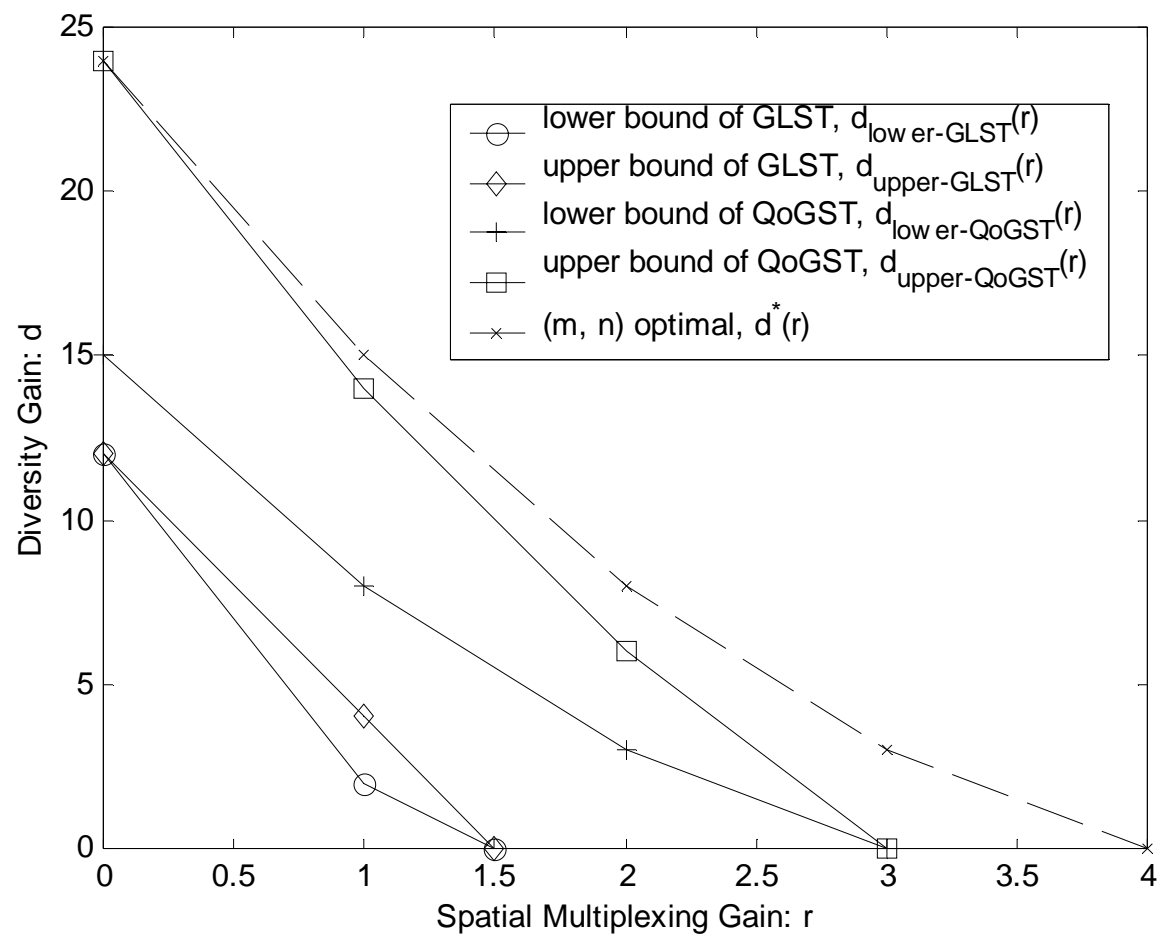

Fig. 6: Diversity-multiplexing tradeoff curves of the upper and lower bounds of GLST, the upper and lower bounds of QoGST and the optimal one when $m=K=6, n=4$. For GLST, $G=2, g_{m}=g_{b}=3$, and $T=4$. For QoGST, $g=3, G_{m}=G_{b}=2$, and $T=2$.

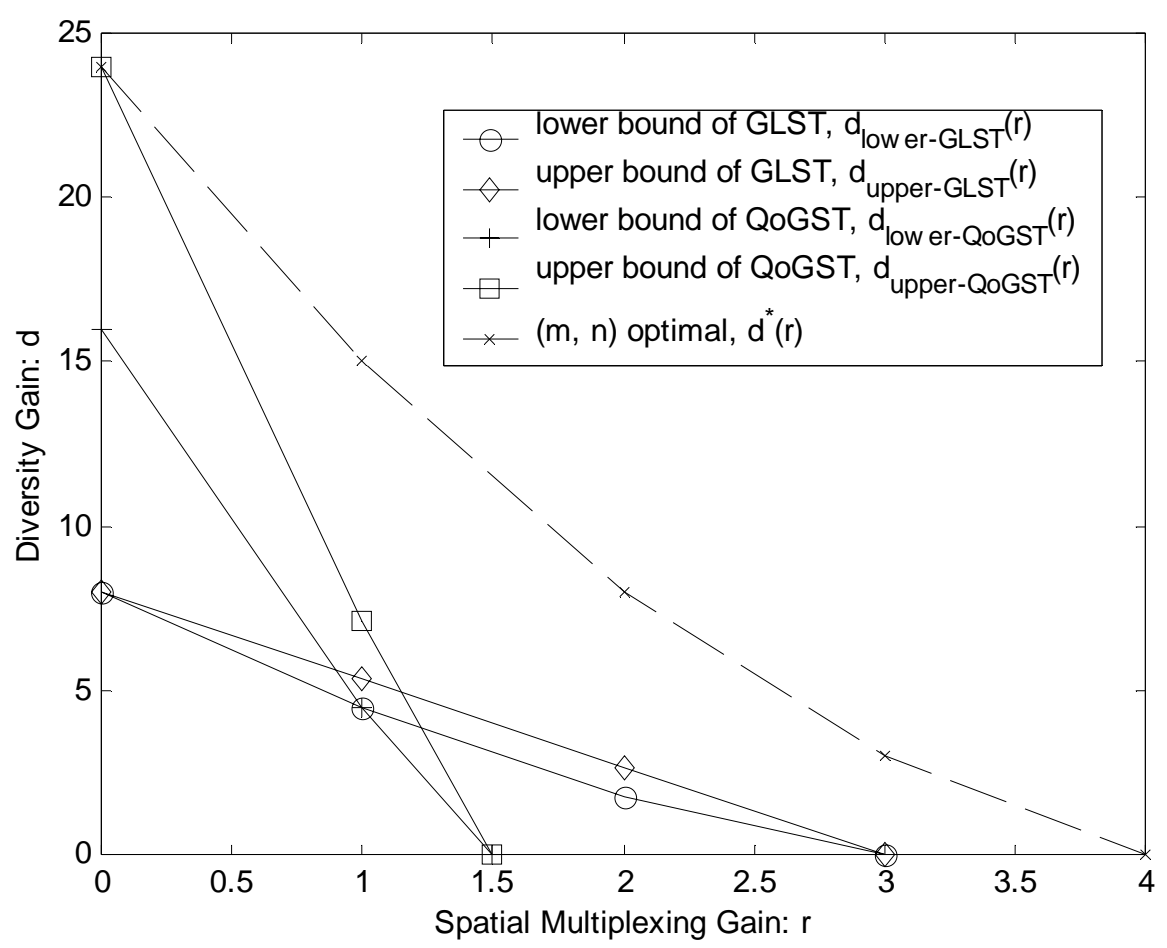

Fig. 7: Diversity-multiplexing tradeoff curves of the upper and lower bounds of GLST, the upper and lower bounds of QoGST and the optimal one when $m=K=6, n=4$. For GLST, $G=3, g_{m}=g_{b}=2$, and $T=2$. For QoGST, $g=2, G_{m}=G_{b}=3$, and $T=4$. 


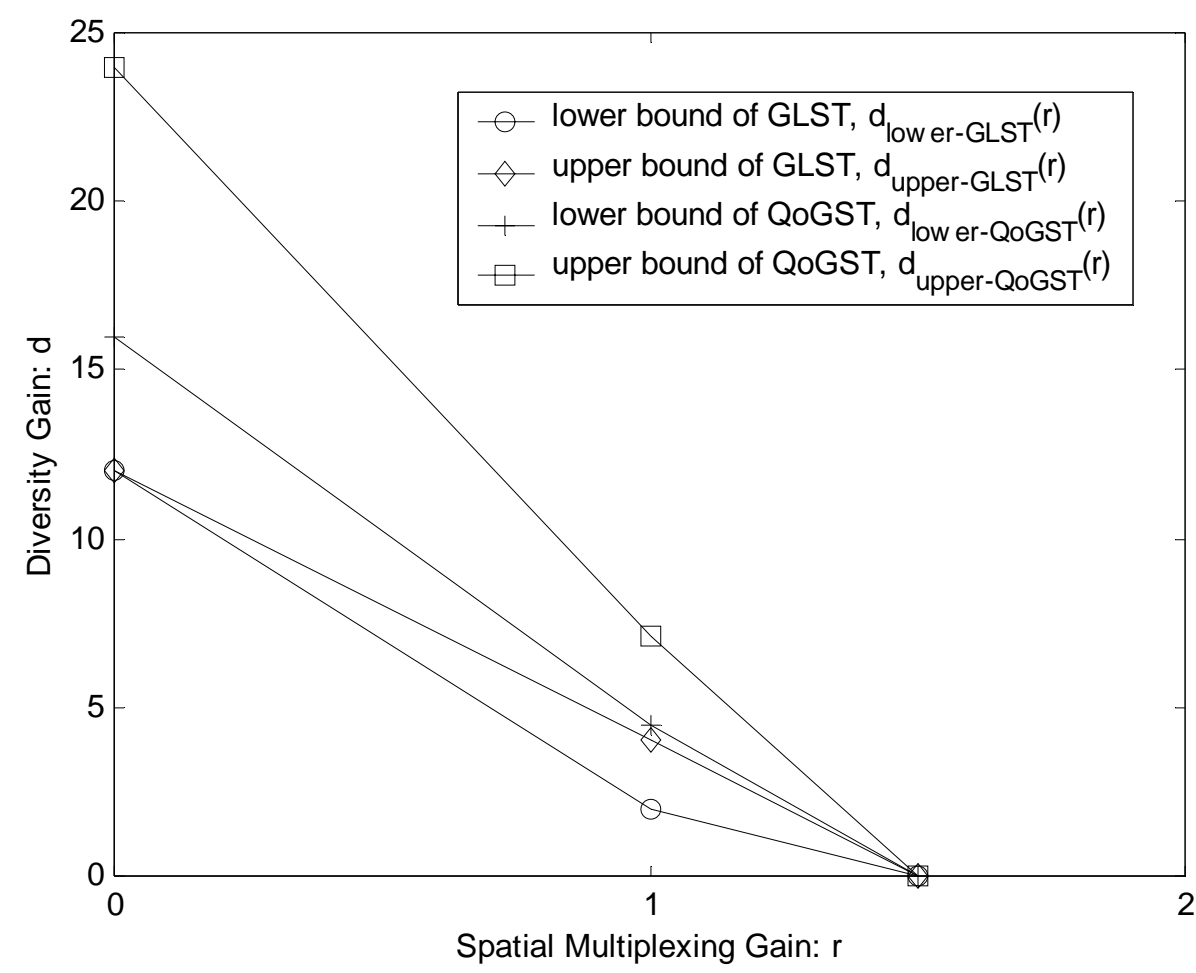

Fig. 8: Diversity-multiplexing tradeoff curves of the upper and lower bounds of GLST, and the upper and lower bounds of QoGST when $m=K=6, n=4$. For GLST, $G=2, g_{m}=g_{b}=3$, and $T=4$. For QoGST, $g=2, G_{m}=G_{b}=3$, and $T=4$.

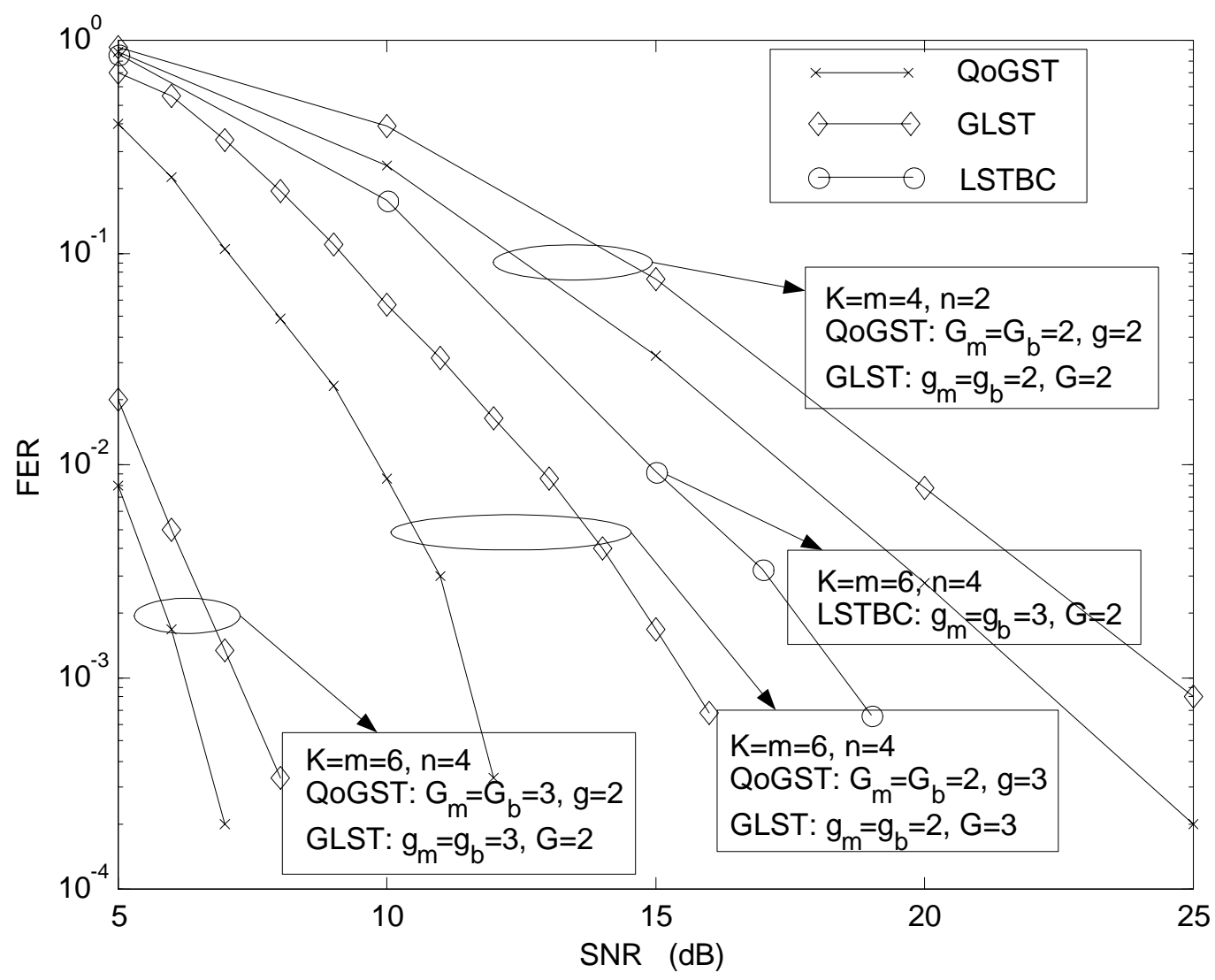

Fig. 9: FER vs. SNR for QoGST, GLST and LSTBC. 